\title{
Aging and the Psychological Refractory Period: Task-Coordination Strategies in Young and Old Adults
}

\author{
Jennifer M. Glass and Eric H. Schumacher \\ University of Michigan
}

Erick J. Lauber
University of Georgia

\author{
Eileen L. Zurbriggen, Leon Gmeindl, David E. Kieras, and David E. Meyer \\ University of Michigan
}

\begin{abstract}
The apparently deleterious effect of aging on dual-task performance is well established, but there is little agreement about the source of this effect. Studies of the psychological refractory period (PRP) indicate that young adults can flexibly control dual-task performance through task-coordination strategies. Thus, the performance of older adults might differ from young adults because older adults use different task-coordination strategies. To test this hypothesis, the executive-process interactive control (EPIC) architecture was applied to quantify the reaction time data from two PRP experiments conducted with young (age 18-26) and older (age 60-70) adults. The results show that participants' ability to coordinate the processing of two tasks did not decline with age. However, dual-task time costs were greater in the older adults. Three sources for this increase were found: generalized slowing, process-specific slowing, and the use of more cautious task-coordination strategies by the older adults.
\end{abstract}

In many situations where optimum performance is crucial, several component tasks are involved. For example, a driver must simultaneously steer, maintain a certain speed, plan a route, and

Jennifer M. Glass, Eric H. Schumacher, Eileen L. Zurbriggen, Leon Gmeindl, and David E. Meyer, Department of Psychology, University of Michigan; Erick J. Lauber, Department of Psychology, University of Georgia; David E. Kieras, Department of Electrical Engineering and Computer Science, University of Michigan.

Jennifer M. Glass is now at the Institute for Social Research and Department of Psychiatry, University of Michigan. Eric H. Schumacher is now at the Department of Psychology, University of California at Berkeley. Eileen L. Zurbriggen is now at the Department of Psychology, University of Oregon.

This research was supported by Grant N00014-92-J-1173 from the U.S. Office of Naval Research to the University of Michigan, David E. Kieras and David E. Meyer, Principal Investigators. Support was also provided by the Center for Applied Cognitive Research on Aging, one of the Edward R. Roybal Centers for Research in Applied Gerontology, Grant 1 P50 AG1175-03 from the National Institute on Aging of the National Institutes of Health. This research is based on a doctoral dissertation by Jennifer $\mathbf{M}$. Glass at the University of Michigan. Portions of this research were first presented at the Cognitive Aging Conference, April 1998, and at the meeting of the Psychonomics Society, November 1998. We thank Alan Hartley for insightful comments on a draft of this article. We also thank past and present members of the Brain, Cognition, and Action Laboratory at the University of Michigan (Elizabeth Emmons, David Fencsik, Cerita Jones, Ryan Kettler, Shane Mueller, Mollie Schweppe, Travis Seymour, and B. J. Woodside) for assistance and helpful suggestions.

Correspondence concerning this article should be addressed to Jennifer M. Glass, Institute for Social Research, University of Michigan, 426 Thompson Street, Ann Arbor, Michigan 48106-1248. Electronic mail may be sent to jglass@umich.edu. react to other cars. In the laboratory, a person's ability to cope with such situations yields data about how information processing is coordinated for the tasks at hand and about the fundamental limits of the human information-processing system. Thus, dual-task performance is an important area of both applied and basic research in psychology. On the basis of such research, it has been well documented that older adults seem to have more difficulty performing successfully in dual-task situations (for reviews, see Hartley, 1992; Kausler, 1991; Kramer \& Larish, 1996; McDowd, Vercruyssen, \& Birren, 1991), yet there has been no consensus about theoretical explanations for this age effect. To help resolve this impasse, we use a computational modeling framework, the executive-process/interactive-control (EPIC) architecture, to provide insights about the effects of age on dual-task performance in the psychological refractory period (PRP) procedure.

\section{Aging and Dual-Task Performance}

In the frequently quoted words of Craik (1977), "one of the clearest results in the experimental psychology of aging is the finding that older subjects are more penalized when they must divide their attention" (p. 391). Research carried out in the intervening 20 years has served to bolster this claim, but there is little agreement about the source of the deficit. The competing theoretical explanations that have been offered thus far can be divided roughly into three distinct, but not mutually exclusive, categories: generalized slowing, specific deficits in dual-task performance, and reduction in processing resources.

\section{Generalized Slowing}

According to the generalized-slowing hypothesis (Bashore, 1994; Cerella, 1985; Salthouse, 1985, 1996) and also the related 
complexity hypothesis (McDowd \& Craik, 1988; McDowd et al., 1991), age-related declines in dual-task performance involve a slowing of all cognitive, perceptual, and motor processes. Therefore, impairment in dual-task performance with age stems from the slowing of performance for the component tasks, as well as from slowing of processes that are necessary to coordinate the component tasks. Both the generalized slowing and complexity hypotheses predict that dual-task costs incurred by older adults should be proportional to dual-task costs of young adults, as found in some studies (e.g., Salthouse, Fristoe, Lineweaver, \& Coon, 1995; Somberg \& Salthouse, 1982). However, several other studies have reported disproportionately large dual-task costs for the elderly (Crossley \& Hiscock, 1992; Park, Smith, Dudley, \& Lafronza, 1989; Ponds, Brouwer, \& van Wolffelaar, 1988; Salthouse, Rogan, \& Prill, 1984). Such findings are not easily explained by generalized slowing. Furthermore, some studies have revealed larger age effects for tasks that are less complex than for tasks that are more complex (e.g., Korteling, 1991). This is at odds with the complexity hypothesis, which predicts larger age effects for more complex tasks.

\section{Specific Age Deficits}

Given the frequent occurrence of disproportionately large dualtask costs for older adults (Hartley, 1992; Kausler, 1991), other explanations have focused on specific deficits beyond generalized slowing. For example, robust age effects have been found in measures of working memory (Salthouse \& Babcock, 1991). Many studies with large age-related dual-task costs used tasks that rely on working memory (e.g., Park et al., 1989; Salthouse et al., 1984), suggesting that declines in working memory may underlie the age-related decline in dual-task performance. However, some studies with little or no working-memory requirements have also found disproportionately large dual-task costs for older participants (e.g., Hawkins et al., 1992; Korteling, 1991).

Recently, West (1996) and Moscovitch and Winocur (1992, 1995) have proposed that many cognitive aging effects are caused by declining frontal lobe function, which hampers the ability to maintain attention and to switch attention between several tasks. Clearly, these are important abilities for successful dual-task performance. Therefore, declines in such executive functions could explain the large dual-task costs associated with age. For example, Allen, Smith, Vires-Collins, and Sperry (1998) argued that there are age-related declines in time-sharing ability. Similarly, N. D. Anderson, Craik, and Naveh-Benjamin (1998) argued that older adults may have difficulty in attentional control over encoding processes, and Salthouse et al. (1984) suggested that older adults are less efficient in allocating attention.

Although these are plausible explanations for disproportionately large effects of age on dual-task performance, it is then problematic to explain the proportionate costs that are sometimes found. One possible solution to this problem would be to claim that the need for executive control was low in the cases where older adults had proportional dual-task costs. However, this claim requires an explicit theory of the executive processes necessary for dual-task performance, which previous theorists have not supplied.

\section{Reduced Processing Resources}

Another explanation for the effects of age on dual-task performance hypothesizes reduced processing resources as the underlying cause. The reduced-resource hypothesis is common to many theories of cognitive aging (Craik \& Byrd, 1982). However, there are many conceptual difficulties associated with resource theories (Allport, 1993; Navon, 1984; Meyer \& Kieras, 1997a, 1999). In addition, reduced-resource hypotheses cannot explain results where more complex or difficult tasks are associated with smaller age effects on dual-task performance than are less complex tasks (e.g., Korteling, 1991).

\section{Task-Coordination Strategies and Aging}

To summarize, none of the previous explanations of age effects on dual-task performance can easily account for diverse cases of proportionate and disproportionately large dual-task costs incurred by older adults. We therefore need to consider an alternative explanation for the conflicting results. Perhaps older adults use different task-coordination strategies than young adults. It is plausible that older adults might use different task-coordination strategies because we know that young adults can spontaneously adopt different strategies in dual-task performance. For example, Damos and Wickens (1980) identified three distinct dual-task strategies in young adults. Recently, we too have reported similar evidence of different task-coordination strategies in young adults (Meyer et al., 1995; Schumacher et al., 1999). These findings could provide a basis to explain why and how dual-task costs are sometimes, but not always, disproportionately larger for older adults.

Perhaps in some test situations, older participants use the same task-coordination strategy as young participants. In this case, the only difference between the participants would be generalized slowing; therefore, the dual-task costs of the older participants would be proportional to the dual-task costs of the young participants. In other situations, older participants may instead adopt a different, less efficient task-coordination strategy than the young participants. In this case, the dual-task costs would be disproportionately large for the older participants. ${ }^{1}$

Others have noted the potential importance of strategies for explaining cognitive aging effects. Welford (1958) observed that older adults often used a different task strategy than did young adults. Salthouse $(1985,1996)$ noted that changes in processing speed might make some strategies ineffective for older adults, so that other, less efficient strategies (compared with those of young adults) have to be adopted instead. Consistent with these considerations, Kramer and Larish (1996) identified research on age differences in dual-task strategies as an important area of further investigation.

This concern about strategies has been applied to dual-task research. For example, Salthouse et al. (1984) used attention

\footnotetext{
1 The finding of proportional dual-task costs is consistent with the inference that young and older adults used the same strategy. However, it is also possible for each age group to use different strategies that happen to yield proportional dual-task costs for the older adults. This highlights the need for explicit theories of task-coordination strategies in order to properly interpret the theoretical importance of relative dual-task costs across young and older adults.
} 
operating characteristics to derive "strategy-independent" measures of divided attention costs. In their experiments, young and old adults had to divide attention between two short-term memory (digits and letters) tasks under conditions with different instructions to emphasize one task over the other. Older adults showed larger decrements in the divided attention conditions, although they were able to vary task emphasis. Salthouse et al. concluded that although older adults can allocate attention, they may be less efficient in this ability. N. D. Anderson et al. (1998) found that older adults were less prone than young adults to vary attentional emphasis between encoding for long-term memory and a fourchoice reaction-time (RT) task. However, the exact nature of the executive control necessary to vary attentional emphasis is not clear. The word "strategy" in Salthouse et al. and Anderson et al. refers to the bias for performing one task over another, but the exact processing steps necessary to coordinate the two tasks, and the ways these steps might change with age, have not been specified.

It seems likely that the conflicting results in the literature will not be explained by a single elementary account; rather, some combination of distinct factors must be at work (for a similar conclusion about the multifactorial nature of divided attention costs, see N. D. Anderson et al., 1998). Therefore, the current work examines specific task-coordination strategies in the context of aging, combined with generalized slowing and possible changes in executive control. The EPIC architecture (Meyer \& Kieras, 1997a, $1997 \mathrm{~b}, 1999$ ) provides an ideal vehicle for analyzing the combined effects of slowing, executive function, and task-coordination strategies because this framework deals explicitly with the sensorymotor, cognitive, and executive processes necessary for dual-task performance.

\section{The Psychological Refractory Period}

To study aging and dual-task performance with EPIC, we tested young and older adults using the PRP procedure (for reviews, see Meyer \& Kieras, 1997a; Pashler, 1994). In this procedure, two tasks are performed on each trial. A trial starts with a warning signal, followed by a stimulus for the first task in which the participant must make a rapid choice reaction. Soon after the onset of the Task 1 stimulus, the stimulus for the second task is presented, and the participant must make another rapid choice reaction to it. The time between the onsets of the two stimuli is the stimulus onset asynchrony (SOA), which can vary from short (0 $\mathrm{ms})$ to long (1,000 ms or more). Participants are instructed to give Task 1 priority over Task 2 . Reaction times are measured to assess how much Task 1 processing interferes with Task 2 processing.

Typical findings from the PRP procedure are shown in Figure 1. The mean Task 2 RTs increase as SOA decreases, manifesting the interference of Task 1 with Task 2 at the shorter SOAs. This interference is called the PRP effect and is a measure of dual-task cost. In contrast, mean Task 1 RTs are typically not affected by SOA, demonstrating that participants obey the instructions to give Task 1 higher priority.

Although Welford (1952) published empirical and theoretical work about the PRP, he apparently never tested older adults with this procedure, despite his other interests in cognitive aging. Recently, however, Allen et al. (1998) used the PRP procedure to examine age differences in dual-task performance. From their

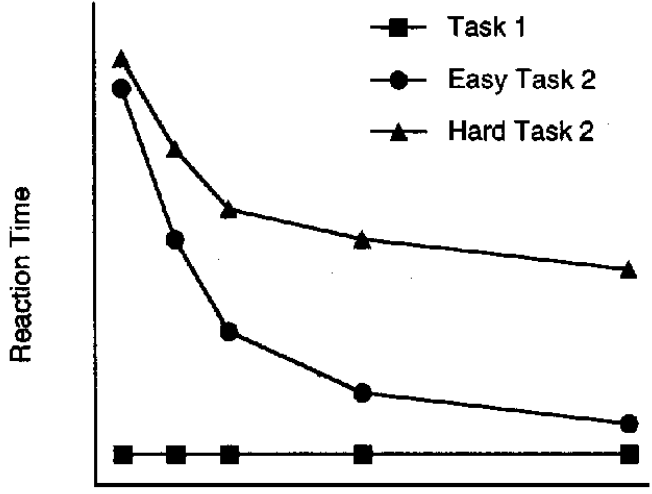

Stimulus Onset Asynchrony

Figure 1. Typical reaction times (RTs) for Task 1 and easy and hard Task 2 from the psychological refractory period (PRP) procedure. Task 1 RTs are not affected by stimulus onset asynchrony (SOA), but Task 2 RTs decrease as SOA increases. The PRP effect is the difference between the Task 2 RT at the shortest SOA and the Task 2 RT at the longest SOA. Here the effect of Task 2 difficulty is smaller at the shortest SOA. Thus, the PRP curves (i.e., the lines representing Task 2 RTs) appear to diverge.

observation of larger PRP effects in the older adults, they concluded that older adults have a time-sharing deficit. Yet there are some reasons to question this conclusion. First, Allen et al.'s participants had very little practice with the tasks, either singly or in the PRP procedure (only 20 trials of dual-task practice). This lack of practice may account for several other observations. For example, participants had extremely long RTs for a two-choice tone discrimination task (e.g., the young adults had a mean RT of approximately $1,400 \mathrm{~ms}$ at the longest $\mathrm{SOA}$ ). Furthermore, participants did not obey the PRP instructions to give Task 1 higher priority, as evidenced by large effects of SOA on Task 1. Again, this likely stems from insufficient practice. Finally, Allen et al. did not consider strategy differences as a source of the age effect. Thus, although the PRP procedure may be used with older adults to obtain theoretically interesting results, we believe that further careful experimentation with this procedure is needed.

Consistent with our belief, Hartley and Little (1999) have also used the PRP procedure to investigate age differences in dual-task performance. In their experiments, they found that older adults had larger PRP effects than younger adults, but only when the responses for each task were in the same motor modality (e.g., manual keypress) and there was pressure to perform both tasks quickly. According to Hartley and Little, these results are not consistent with a reduced-resource model. Instead, they concluded that the ability to manage the tasks was intact in older adults, even though the older adults were somewhat slowed.

As a result, the existing research on age differences measured with the PRP procedure has produced conflicting conclusions about the source of the age effect. The apparent conflict may stem from the lack of well-specified theories for characterizing dualtask performance. To fill this void, we used the EPIC architecture as a theoretical basis for analyzing and understanding diverse systematic age differences in dual-task performance under the PRP procedure. The major features of the EPIC architecture and our 
computational models of dual-task performance are described next.

\section{The EPIC Architecture and Adaptive Executive-Control Models}

EPIC is a cognitive architecture that provides precise computational models of human multiple-task performance in both laboratory and applied settings (Meyer \& Kieras, 1997a, 1997b, 1999). These models are achieved through EPIC's explicit specifications of cognitive, sensory, and motor processes. Furthermore, on the basis of EPIC, the interactions of cognitive, sensory, and motor processes during multiple-task performance are detailed with formal hypotheses about supervisory executive processes and taskcoordinating strategies. Thus, EPIC is ideally suited for studying aging, executive control, and dual-task strategies.

The EPIC architecture consists of interconnected LISP software modules for symbolic perceptual-, motor-, and cognitiveinformation processing. These modules are designed to emulate basic components of the human information-processing system. In particular, EPIC has three main types of component: perceptual processors, motor processors, and a cognitive processor, which are illustrated in Figure 2. The perceptual processors include visual, auditory, and tactile ones. The motor processors include manual, ocular, and vocal ones. The cognitive processor contains working- memory stores and a production rule interpreter, which enables executive control and task procedures to be represented by sets of production (if condition, then action) rules.

An important feature of the cognitive processor is that it operates in cycles. On each processing cycle, the production rule interpreter tests the conditions for all of the production rules in EPIC's procedural memory. The production rule interpreter then executes the actions for all of the rules whose conditions match the contents of working memory. The mean duration of the cognitive processor cycle is $50 \mathrm{~ms}$, based on observations of periodicity in human information processing (see Meyer \& Kieras, 1997a). The duration of the cognitive-processor cycles does not depend on the number of production rules to be tested, and there is no set limit on the number of rules that can be tested on any given cycle. Thus, there is no central hardware decision or response-selection bottleneck in EPIC.

This can be contrasted with the response-selection bottleneck (RSB) hypothesis that has been proposed to explain the results of PRP experiments (e.g., De Jong, 1993; McCann \& Johnston, 1992; Pashler, 1984, 1994; Pashler \& Johnston, 1989; Welford, 1952). The RSB hypothesis states that there is an information-processing stage that selects a response to be made for a stimulus, and that this stage can select only one response at a time. Thus, according to the RSB hypothesis, if two tasks are underway at the same time, the

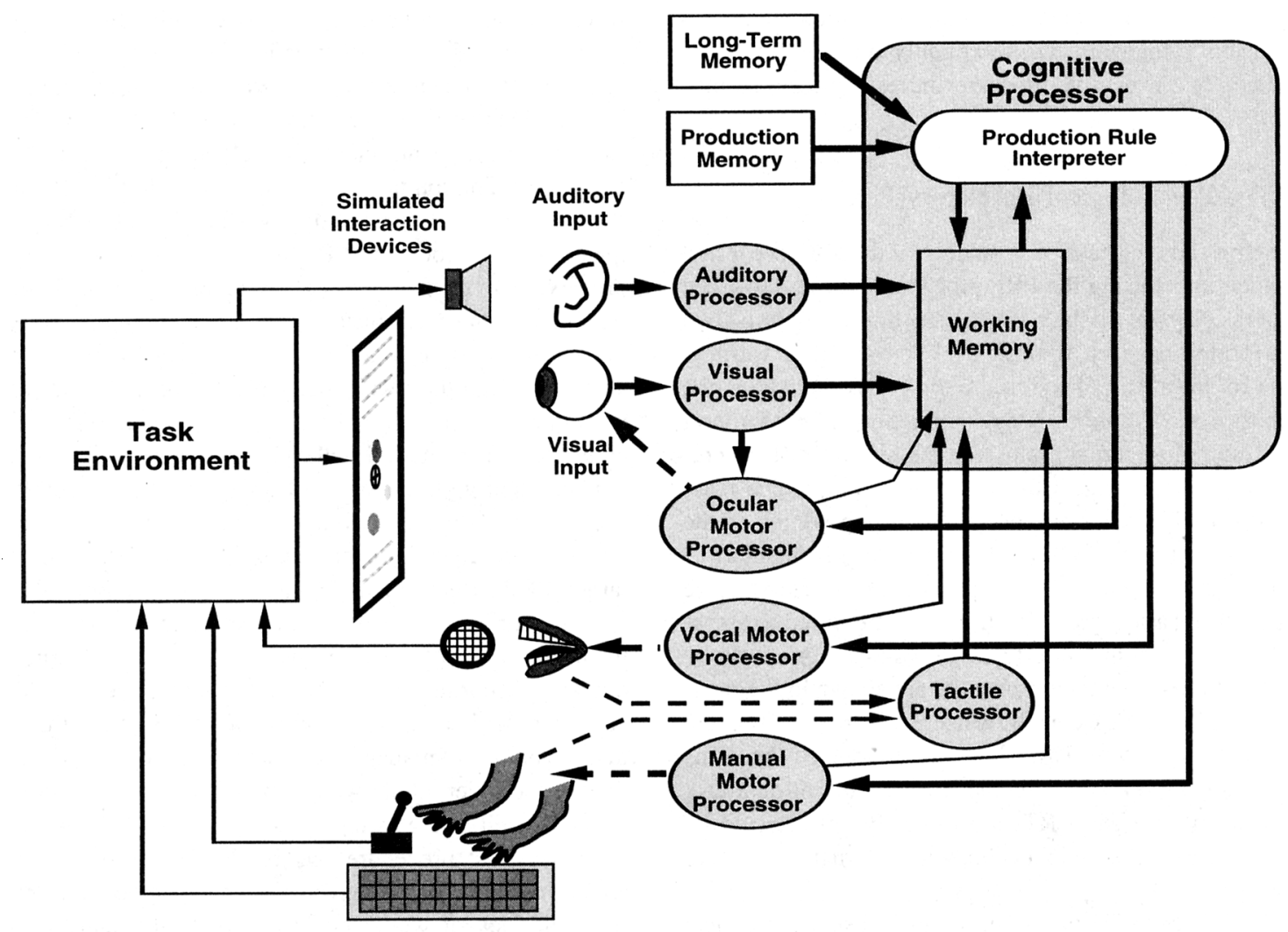

Figure 2. Overview of the executive-process/interactive-control (EPIC) architecture. From "A Computational Theory of Executive Cognitive Processes and Multiple-Task Performance: Part 2. Accounts of Psychological Refractory-Period Phenomenon," by D. E. Meyer and D. E. Kieras, 1997, Psychological Review, 104, p. 750. Copyright 1997 by the American Psychological Association. Reprinted with permission of the author. 
selection of a response for one of the tasks will have to wait until response selection for the other task is completed.

EPIC's omission of a cognitive-processing bottleneck is based on some fundamental metatheoretical principles (Meyer \& Kieras, 1997a, 1999). First, EPIC is an integrated information-processing architecture that incorporates known characteristics of human information processing, in the spirit of other work on unified theories of cognition (J. R. Anderson, 1993; Newell, 1990). Second, the omission of a cognitive bottleneck allows EPIC to avoid pitfalls of vagueness and circularity inherent in limited resource and singlechannel theories (Allport, 1993; Navon, 1984). This is especially important for application to cognitive aging, where resource theories are popular despite their inherent pitfalls. Third, EPIC embodies the constraints imposed by the human perceptual-motor information-processing system, thus enabling veridical accounts of dual-task decrements that otherwise might be misattributed to a cognitive bottleneck. Finally, and perhaps most importantly, because EPIC does not include a single-channel, central cognitive bottleneck, it fosters strong emphasis on flexible executive strategies for multiple-task performance that people presumably use to satisfy situational constraints.

Given this latter emphasis, an important step in building an EPIC model of multiple-task performance is to characterize and specify executive processes that coordinate the processing of component tasks. Meyer and Kieras (1997a, 1997b) have developed a class of adaptive executive-control (AEC) models for performance in situations where one task has priority over another task, as in the PRP procedure. In the AEC models (Figure 3), the executive processes control the timing of secondary task-processing stages, thereby determining the degree of temporal overlap between the two tasks and ensuring the primacy of Task 1 . The functions performed by the executive processes include (a) allowing primary and secondary task processes to begin at the onset of a trial; (b) specifying a Task 2 lockout point; (c) specifying a Task 1 unlocking event; (d) waiting for the unlocking event to occur; and (e) unlocking Task 2 processes so that Task 2 can be completed after the Task 1 unlocking event occurs. For understanding performance in the PRP procedure, the executive processes that specify the Task 2 lockout point and the Task 1 unlocking event are most important.

The Task 2 lockout point represents the point in the course of Task 2 where processing is stopped temporarily, ensuring that the response for Task 1 can be made before the response for Task 2 . The placement of the Task 2 lockout point influences the potential amount of temporal overlap between Task 1 and Task 2 processes. An early Task 2 lockout point (e.g., before the Task 2 response-

\section{Task 1 Processes}

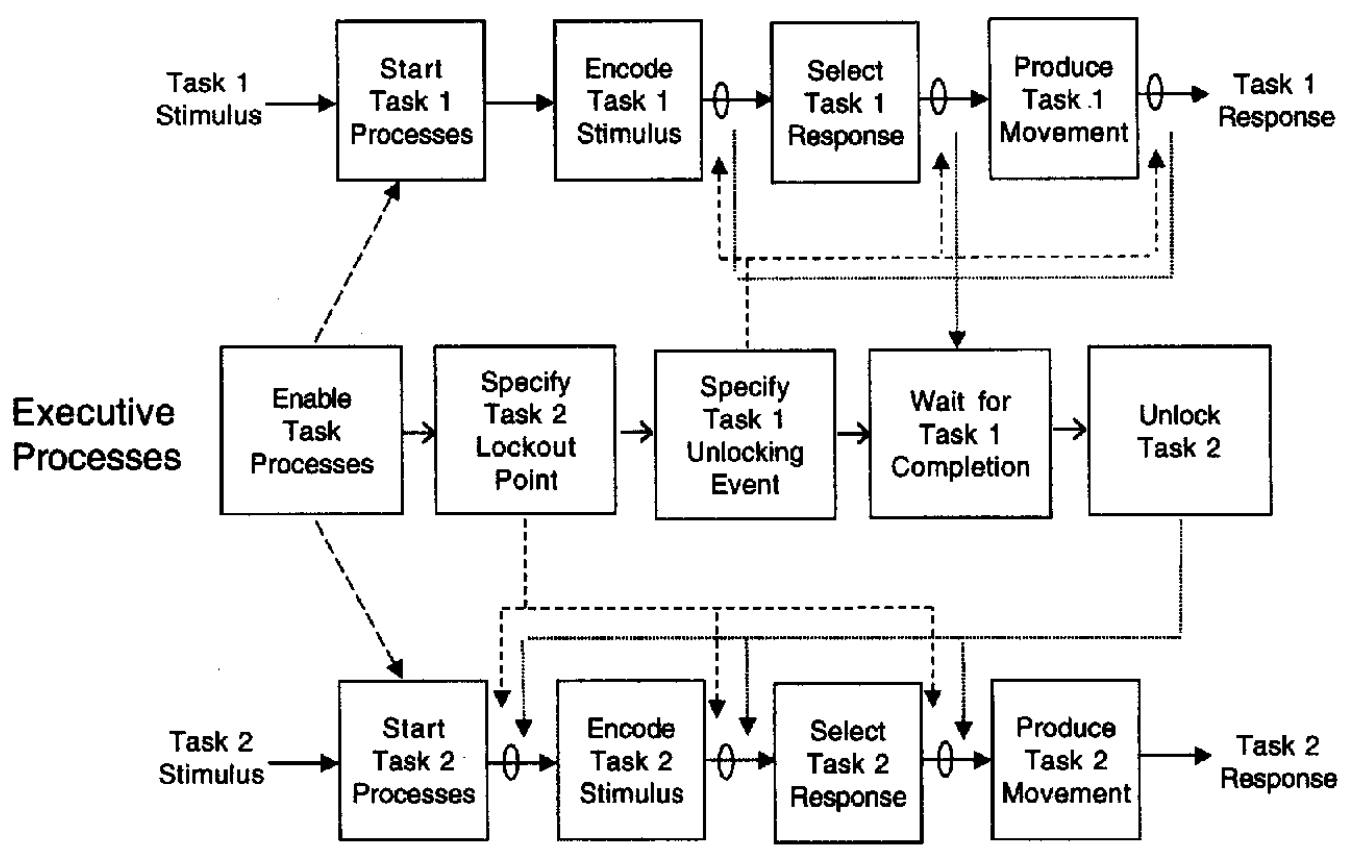

\section{Task 2 Processes}

Figure 3. Component processes for adaptive executive-control (AEC) models whereby tasks in the psychological refractory period (PRP) procedure may be flexibly scheduled. The top row depicts the Task 1 processes, the bottom row depicts Task 2 processes, and the middle row depicts the executive processes involved in coordinating the two tasks. Arrows leading to and from various executive processes denote where a Task 2 lockout point may be set, where a Task 1 unlocking event may occur, where processing for Task 1 is deemed to be completed, and where processing for Task 2 should be resumed. From "Concurrent Response-Selection Processes in Dual-Task Performance: Evidence for Adaptive Executive Control Over Task-Scheduling Strategies," by E. H. Schumacher, E. J. Lauber, J. M. Glass, E. L. Zurbriggen, L. Gmeindl, D. E. Kieras, and D. E. Meyer (1999), Journal of Experimental Psychology: Human Perception and Performance, 25, p. 793. Copyright 1999 by the American Psychological Association. Reprinted with permission of the author. 
selection stage) will result in less temporal overlap between the two tasks. Conversely, a late Task 2 lockout point (i.e., after the Task 2 response-selection stage) will result in more overlap between the two tasks.

The Task 1 unlocking event also influences the amount of temporal overlap between Task 1 and Task 2 processes. When the Task 1 unlocking event occurs, it signifies that Task 1 is sufficiently complete and steps can be taken to resume Task 2 processing. Thus, if the specified Task 1 unlocking event occurs relatively early (e.g., at the end of Task 1 stimulus identification), then there can be considerable temporal overlap in the remaining processes of both tasks. Conversely, if the Task 1 unlocking event occurs relatively late (e.g., at the end of Task 1 movement production), then there will be less temporal overlap in the remaining processes of the two tasks.

The AEC models can account for many observed PRP phenomena, including ones that are not predicted by the response-selection bottleneck hypothesis. The PRP effect is explained as a result of satisfying the instructions that Task 1 should have priority over Task 2 (Meyer \& Kieras, 1997a; Schumacher et al., 1999). If Task 2 processing were allowed to proceed entirely without interruption, it might finish first, causing frequent responses that violate the instructions to give Task 1 priority. To avoid this, a Task 2 lockout point and a Task 1 unlocking event must be specified, and they will delay Task 2 processing at the short SOAs, giving rise to the PRP effect. As explained before, when the Task 2 lockout point occurs late in Task 2 processing and the Task 1 unlocking event occurs early in Task 1 processing, there will be opportunity for substantial temporal overlap in the processes for both tasks. This will yield a small PRP effect. If on the other hand, the Task 2 lockout point occurs early and the Task 1 unlocking event occurs late, then there will be less opportunity for temporal overlap between the two tasks. This will yield a large PRP effect.

Another aspect of PRP data that can be explained by the AEC models is the shape of the Task 2 PRP curves when the difficulty of Task 2 response selection has been manipulated. The shape of the Task 2 PRP curves is influenced by the location of the Task 2 lockout point. At short SOAs, the lockout of Task 2 introduces a period where no processing for Task 2 occurs. If the Task 2 lockout point is early (i.e., before the Task 2 response-selection stage), then the period of "processing slack" (De Jong, 1993; McCann \& Johnston, 1992; Pashler, 1984; Schumacher et al., 1999) also occurs before the Task 2 response-selection stage. In this case, the early Task 2 lockout point mimics a responseselection bottleneck. For example, the top panel of Figure 4 shows the prediction based on an early Task 2 lockout point. Because the processing slack occurs before the response-selection stage, the effect of response-selection difficulty is the same for all SOAs, and the PRP curves will be parallel (that is, SOA and Task 2 responseselection difficulty will have additive effects). Several studies have reported additive effects of Task 2 response-selection difficulty and SOA (e.g., McCann \& Johnston, 1992; Pashler, 1984, 1994).

In contrast, if the Task 2 lockout point is a late one (i.e., after Task 2 response selection), then the effect of Task 2 responseselection difficulty will not be the same for all SOAs (illustrated in the bottom half of Figure 4). This prediction stems from processing slack that occurs after the response-selection stage when the SOA is short. The difference between the easy Task 2 and the hard Task 2 is masked by the presence of processing slack, and the effect of Task 2 response-selection difficulty is reduced at the shortest SOA, leading to diverging (i.e., underadditive) PRP curves. An example of diverging PRP curves corresponding to task coordination with a late Task 2 lockout point, is illustrated in Figure 1. Diverging PRP curves have been reported by Hawkins, Rodriguez, and Reicher (1979); Ivry, Franz, Kingstone, and Johnston (1994); Karlin and Kestenbaum (1968); Meyer et al. (1995); and Schumacher et al. (1999).

In summary, according to the AEC models, several taskcoordination strategies are possible under the PRP procedure. One factor that should influence the selection of a task-coordination strategy is the relative difficulty of Task 1 compared to Task 2 . If Task 1 is relatively easy, and there is little chance of Task 2 finishing before Task 1 , then a task-coordination strategy that allows a lot of overlap between processes for both tasks is best because it minimizes response times for Task 2 while still protecting the primacy of Task 1 . This "daring" task-coordination strategy is achieved by setting a late Task 2 lockout point, resulting in underadditive PRP curves when Task 2 response-selection difficulty is manipulated.

On the other hand, if Task 1 is hard relative to Task 2, then the participant must cope with the potential problem that the response for Task 2 might be ready before the response for Task 1, especially when the SOA is short. In this case, the task-coordination strategy may tend to be conservative and may not allow as much overlap between the processes for both tasks. This is achieved by setting an early Task 2 lockout point, resulting in additive PRP curves and larger PRP effects than with a daring task-coordination strategy.

\section{Modeling Effects of Aging With the EPIC Architecture}

In our literature review, we described four different theoretical accounts of the effects of age on dual-task performance: generalized slowing, specific deficits in executive control for dual-task performance, reduced cognitive resources, and task-coordination strategy differences. The EPIC architecture can be used to evaluate these explanations. For example, generalized slowing can be modeled in EPIC by increasing the mean cognitive-processor cycle duration. The "young" version of EPIC has a mean cognitiveprocessor cycle duration of $50 \mathrm{~ms}$ (Meyer \& Kieras, 1997a). To model the slower reaction time performance of older adults, we increase the mean cognitive-processor cycle duration by $12 \%$ to 56 $\mathrm{ms}$. Although this specific value might seem arbitrary, it was chosen on several principled bases, which take account of older adults' performance under certain basic RT conditions. Specifically, Somberg and Salthouse (1982) found that simple RTs were about $12 \%$ longer for older adults. Baron and Matilla (1989) found that choice RTs after extensive practice were about $12 \%$ longer for older adults. Finally, the mean period of alpha rhythms in older adults, aged 60 to 65 years, is about $12 \%$ longer than in young adults (Woodruff, 1975; Woodruff-Pak, 1997). These findings provide a plausible estimate for the mean cognitive-processor cycle duration of older adults because they manifest the essential minimum amount of cognitive slowing caused by aging, and because they are least likely to be inflated by additional strategy differences that would increase the apparent amount of slowing beyond that which stems from the "hardware" of the cognitive processors per se. 


\begin{tabular}{|c|c|c|c|c|c|}
\hline Task 1 & \begin{tabular}{|l|} 
Stimulus \\
Encoding
\end{tabular} & $\begin{array}{l}\text { Response } \\
\text { Selection }\end{array}$ & $\begin{array}{l}\text { Response } \\
\text { Production }\end{array}$ & & \\
\hline $\begin{array}{l}\text { Easy Task } 2 \\
\text { Short SOA }\end{array}$ & \begin{tabular}{|l|l|} 
SOA & $\begin{array}{l}\text { Stimulus } \\
\text { Encoding }\end{array}$ \\
\end{tabular} & Slack & $\begin{array}{l}\text { Response } \\
\text { Selection }\end{array}$ & $\begin{array}{l}\text { Response } \\
\text { Production }\end{array}$ & Iftury 5 \\
\hline $\begin{array}{l}\text { Hard Task } 2 \\
\text { Short SOA }\end{array}$ & \begin{tabular}{|l|l|} 
SOA & Stimulus \\
& Encoding \\
\end{tabular} & Slack & $\begin{array}{l}\text { Response } \\
\text { Selection }\end{array}$ & & $\begin{array}{l}\text { Response } \\
\text { Production }\end{array}$ \\
\hline $\begin{array}{l}\text { Easy Task } 2 \\
\text { Long SOA }\end{array}$ & SOA & $\begin{array}{l}\text { Stimulus } \\
\text { Encoding }\end{array}$ & $\begin{array}{l}\text { Response } \\
\text { Selection }\end{array}$ & \begin{tabular}{|l} 
Response \\
Production
\end{tabular} & \\
\hline $\begin{array}{l}\text { Hard Task } 2 \\
\text { Long SOA }\end{array}$ & SOA & $\begin{array}{l}\text { Stimulus } \\
\text { Encoding }\end{array}$ & $\begin{array}{l}\text { Response } \\
\text { Selection }\end{array}$ & & $\begin{array}{l}\text { Response } \\
\text { Production }\end{array}$ \\
\hline
\end{tabular}

\section{Early Task 2 Lockout Point}

\begin{tabular}{|c|c|c|c|c|c|}
\hline Task 1 & $\begin{array}{l}\text { Stimulus } \\
\text { Encoding }\end{array}$ & $\begin{array}{l}\text { Response } \\
\text { Selection }\end{array}$ & $\begin{array}{l}\text { Response } \\
\text { Execution }\end{array}$ & & \\
\hline $\begin{array}{c}\text { Easy Task } 2 \\
\text { Short SOA }\end{array}$ & \begin{tabular}{|l|l|} 
SOA & $\begin{array}{l}\text { Stimulus } \\
\\
\text { Encoding }\end{array}$ \\
\end{tabular} & $\begin{array}{l}\text { Response } \\
\text { Selection }\end{array}$ & Slack & $\begin{array}{l}\text { Response } \\
\text { Execution }\end{array}$ & $\begin{array}{l}1 \\
1 \\
1\end{array}$ \\
\hline $\begin{array}{c}\text { Hard Task } 2 \\
\text { Short SOA }\end{array}$ & \begin{tabular}{|l|l|} 
SOA & $\begin{array}{l}\text { Stimulus } \\
\text { Encoding }\end{array}$ \\
\end{tabular} & $\begin{array}{l}\text { Response } \\
\text { Selection }\end{array}$ & & $\begin{array}{l}\text { Response } \\
\text { Execution }\end{array}$ & \\
\hline $\begin{array}{c}\text { Easy Task } 2 \\
\text { Long SOA }\end{array}$ & SOA & $\begin{array}{l}\text { Stimulus } \\
\text { Encoding }\end{array}$ & $\begin{array}{l}\text { Response } \\
\text { Selection }\end{array}$ & $\begin{array}{l}\text { Response } \\
\text { Execution }\end{array}$ & \\
\hline $\begin{array}{l}\text { Hard Task } 2 \\
\text { Long SOA }\end{array}$ & SOA & \begin{tabular}{|l|} 
Stimulus \\
Encoding
\end{tabular} & $\begin{array}{l}\text { Response } \\
\text { Selection }\end{array}$ & & \begin{tabular}{|l} 
Response \\
Execution
\end{tabular} \\
\hline
\end{tabular}

\section{Late Task 2 Lockout Point}

Figure 4. The role of the locus of slack for the interaction of Task 2 response-selection difficulty and stimulus onset asynchrony (SOA). The top panel illustrates how an early Task 2 lockout point leads to additive effects of Task 2 difficulty and SOA (resulting in parallel psychological refractory period [PRP] curves). The bottom panel illustrates how a late Task 2 lockout point leads to an interaction between Task 2 difficulty and SOA (resulting in diverging PRP curves).

EPIC can also be used to characterize potential deficits in executive control processes. Because EPIC achieves dual-task performance through executive processes that implement taskcoordination strategies, our predictions about the consequences of deficient executive control are more explicit than has been possible previously. In particular, there are two distinct alternative consequences. First, deficient executive control may entail an inability to enforce a task-coordination strategy that ensures Task 1 primacy. This would lead older adults to produce frequent out-oforder responses, especially at the shorter SOAs. Without executive control, there would be no Task 2 lockout point, and each task would run to completion without delays. Thus, consideration of the executive functions involved in dual-task performance leads to the counter-intuitive prediction of smaller PRP effects in the elderly when executive control is deficient, as well as frequent out-oforder responses. Another possible consequence of deficient executive control is that older adults may realize they are unable to follow the PRP instructions easily, and therefore they may adopt an extremely conservative strategy to cope with their inefficient executive control. An example of such a strategy would be shutting the eyes to avoid seeing the Task 2 stimulus until Task 1 is completed. This would result in very large PRP effects for the elderly, with no evidence of overlapping response-selection stages.

In contrast to such extreme strategies that would indicate deficient executive control, EPIC can be used to represent more subtle differences in strategies, by applying the AEC models to describe PRP performance. For example, older participants may have moderately (rather than extremely) more conservative task- 
coordination strategies compared to the more daring strategies of young participants. However, in this context, conservative does not mean deficient. An important insight from the AEC models of PRP performance is that more conservative strategies involve at least as much executive control as do daring strategies. We expect that older adults might be more conservative because this is consistent with previous evidence that older adults frequently have larger dual-task costs than do young adults. In addition, Welford (1958) observed that older men in his studies spent more time planning a move and then spent less time correcting their moves. Smith and Brewer (1995) found that older adults were more careful in avoiding errors, and when they did make errors, they slowed down more than young adults did in response to those errors. This apparent concern about errors may lead older adults to use a taskcoordination strategy that will minimize erroneous out-of-order responses in the PRP procedure. According to the AEC models, such a strategy involves specifying an earlier Task 2 lockout point, a later Task 1 unlocking event, or both.

The fourth explanation of age effects in dual-task performance is a reduction in processing resources. As pointed out earlier, many theorists have discussed the limitations of resource-based explanations of behavior in dual-task situations. Because EPIC does not assume a cognitive bottleneck in executing production rules, the extent to which we can account for PRP performance in older adults will provide at least an existence proof that limited resources are not necessary for explaining age effects in dual-task performance. In addition, we will be able to specify the nature of the dual-task deficit with more precision than is possible by simply appealing to reduced resources.

In summary, there are several hypotheses to be tested here. One hypothesis is that generalized slowing alone will explain the effects of aging in the present two experiments. This slowing will be modeled in EPIC by increasing the cognitive-processor cycle duration. Another hypothesis is that generalized slowing must be paired with more conservative task-coordination strategies in order to account for the older adults' PRP data. This could be accomplished by using an earlier Task 2 lockout point or a later Task 1 unlocking event. If older adults do indeed have more conservative strategies, then their dual-task costs (the PRP effect) will be larger than predicted by generalized slowing alone, and if their Task 2 lockout point occurs before the Task 2 response-selection stage, then they will have additive PRP curves.

To examine possible strategy differences, the difficulty of Task 1 is manipulated across our Experiments 1 and 2. In young adults, a relatively easy Task 1 is more likely to induce daring strategies, whereas a relatively difficult Task 1 is more likely to induce conservative strategies (Meyer et al., 1995). We predict that older adults will not be uniformly conservative across experiments, consistent with previous observations of both proportional and disproportionately large dual-task costs incurred by older adults. However, conservative strategies should not be equated with a lack of executive control. Instead, if older adults have impaired executive control, then we predict either extensive overlap in task processing without any control over Task 2 processes (including increased out-of-order responses and small PRP effects) or use of more extreme strategies that preclude any overlap in the processes for each task.

Therefore, to test the predictions outlined above, two PRP experiments with respectively easy or hard versions of Task 1 are reported. Each experiment uses an auditory-manual Task 1 and a visual-manual Task 2 . Task 1 involves tone discrimination and Task 2 involves digit classification. The response-selection difficulty of Task 2 is manipulated in each experiment, and participants receive two sessions of practice, to ensure their familiarity with the tasks and the PRP instructions.

\section{Experiment 1}

For Task 1 of this experiment, one or the other of two tones was presented on each trial. Speeded responses to the tones were made with either the left index or left middle fingers, depending on their frequencies. For Task 2, one of two printed digits (easy Task 2) or one of eight printed digits (hard Task 2) was presented on each trial. Speeded responses to the digits were made with either the right index or the right middle finger, depending on the digit's identity.

Schumacher et al. (1999) found that under these conditions, young adults spontaneously adopted a strategy that overlapped the response-selection processing stages for Tasks 1 and 2. This daring task-coordination strategy was evident from the shapes of the PRP curves, which diverged as SOA increased and occurred consistently across participants. The present experiment tests whether older adults adopt the same kind of daring strategy as do young adults.

Shown in Table 1 are our predictions about the shapes of the older adults' PRP curves, depending on their task-coordination strategies. If the older adults use the same daring strategy as do the young adults, then the shapes of their PRP curves should be very similar to those of the young adults, but they may involve longer RTs at each SOA because of generalized slowing. However, if older adults are more cautious and use a strategy that precludes overlapping response-selection processes, then their PRP curves should be parallel, and the Task 2 difficulty effect will be the same at each SOA. For example, this could occur by setting the Task 2 lockout point to be earlier than in the young adult's strategy (see Figure 3).

It is also possible that the older adults will be more cautious for only the hard Task 2 . This would lead to a pattern of data in which their PRP curves converge as SOA increases, with the difficulty effect being largest at the shortest SOA (i.e., overadditive PRP curves). Another possibility is that the older adults may adopt a more cautious strategy by selecting a later Task 1 unlocking event, while selecting a Task 2 lockout point that is similar to the young adults. In this case, the diverging shape of the PRP curves will be maintained, but the size of the PRP effect will be larger (i.e., a bigger difference in mean RT between the shortest and longest SOA). Finally, if the older adults cannot enforce any kind of task scheduling, then processing for each task is expected to proceed as soon as its stimulus appears. Very small PRP curves would result from this lack of executive control, because there would be no lockout of Task 2 processing, but many out-of-order responses would also occur at the shortest SOAs.

\section{Method}

\section{Participants}

Ten right-handed older adults ( 5 male and 5 female), aged 60-70 years with a mean age of 65 years, were recruited from the University of 
Table 1

Predicted Patterns of Psychological Refractory Period (PRP) Curves for Older Adults Depending on Their Task-Coordination Strategy

\begin{tabular}{|c|c|c|c|}
\hline Strategy & Task 2 lockout point & Task 1 unlocking event & PRP curves \\
\hline Daring-daring & Same as young adults' & Same as young adults' & $\begin{array}{l}\text { Diverging; PRP effect proportional } \\
\text { to young adults' }\end{array}$ \\
\hline Cautious-daring & $\begin{array}{l}\text { Earlier than young } \\
\text { adults' }\end{array}$ & Same as young adults' & $\begin{array}{l}\text { Parallel; PRP effect } \\
\text { disproportional to young adults' }\end{array}$ \\
\hline Daring-cautious & Same as young adults' & $\begin{array}{l}\text { Later than young } \\
\text { adults' }\end{array}$ & $\begin{array}{l}\text { Diverging; PRP effect } \\
\text { disproportional to young adults' }\end{array}$ \\
\hline Cautious-cautious & $\begin{array}{l}\text { Earlier than young } \\
\text { adults' }\end{array}$ & $\begin{array}{l}\text { Later than young } \\
\text { adults' }\end{array}$ & $\begin{array}{l}\text { Parallel; PRP effect } \\
\text { disproportional to young adults' }\end{array}$ \\
\hline
\end{tabular}

Note. Each alternative strategy is defined by combining a daring (late) or cautious (early) Task 2 lockout point with a daring (early) or cautious (late) Task 1 unlocking event in the executive processes of the adaptive executive-control models.

Michigan Geriatrics Center human participants core. No participants had neurological (e.g., strokes or seizures) or psychiatric (e.g., depression) disorders. This was assessed through information provided by the Geriatrics Center and initial phone contact. Participants reported themselves as having either good, very good, or excellent health. The modal response was very good. There were no responses less than good. The participants had normal or corrected-to-normal vision and did not require hearing aids. Their level of education was quite high. Two were high school graduates, one was a college graduate, and the remainder had at least master's degrees. Participants were paid an hourly rate plus bonuses on the basis of good performance.

Ten right-handed young adult participants ( 8 male and 2 female), aged 18-26 years with a mean age of 20.4 years, were recruited from the University of Michigan campus. They had normal or corrected-to-normal vision, and they were paid an hourly rate plus bonuses on the basis of good performance. The testing of these participants has been reported previously by Schumacher et al. (1999, Experiment 3).

\section{Apparatus}

Stimulus presentation and data collection were controlled by an AST Premium 386 microcomputer. The visual stimuli were presented on a Zenith monochrome monitor. The auditory stimuli were presented over Sennheiser HMD 224 headphones. Responses were made on a specially constructed keyboard, with response keys for each finger of each hand.

Testing was conducted in a dimly lit room. Each older participant sat at a distance of approximately $60 \mathrm{~cm}$ from the display monitor; the distance for young participants was $80 \mathrm{~cm}$.

\section{Stimuli and Responses}

Participants made responses to two stimuli on each trial (a tone and a digit). For Task 1, the tones were $1,200 \mathrm{~Hz}$ or $800 \mathrm{~Hz}$, and responses were keypresses by the left middle finger or the left index finger, respectively. For Task 2, the presentation of one of eight digits (2 through 9 ) required a particular right-hand finger response. If a $2,5,6$, or 9 appeared, then the participant responded with a keypress by the right index finger. If a $3,4,7$, or 8 appeared, then the participant responded with a keypress by the right middle finger. There were two mappings, easy and hard, for the secondary digit task. The hard mapping used all eight digits as possible stimuli, whereas the easy mapping used just two digits ( 2 and 3 ) as possible stimuli. The mappings were varied between blocks.
The Task 1 tone was always presented before the Task 2 digit, and participants had to respond to the tone first. Stimulus onset asynchrony, the time between the onset of the tone and the onset of the digit, varied from trial to trial within a block. There were five different SOAs $(50,150,250$, 500 , or $1,000 \mathrm{~ms}$ ). The particular SOA, tone, and digit for each trial were selected randomly from a set of SOA, tone, and digit combinations predetermined for each block.

\section{Procedure}

The SOA and task combinations were counterbalanced to ensure that each possible combination occurred an equal number of times. There were three sessions, conducted on separate days within 1 week. Each session lasted about $1.5 \mathrm{hr}$. Participants were tested individually.

Participants received written instructions describing the tasks and the point payoff structure. The instructions stated that the response to the auditory task (Task 1) must come before the response to the visual task (Task 2). After the participant read the instructions, the experimenter gave a verbal description of the tasks to ensure that the participant understood the instructions.

Points. The point system was arranged such that the older participants would receive point totals comparable to those of the young participants. The young participants received 200 points ( 300 for older participants) for a correct Task 1 response, minus 2 points for every $10 \mathrm{~ms}$ of reaction time to Task 1; young participants received 100 points ( 150 for older participants) for a correct Task 2 response, minus 1 point for every $10 \mathrm{~ms}$ of reaction time to Task 2 . For young participants, an incorrect response to Task 1 resulted in minus 200 points (300 for older participants), and an incorrect response to Task 2 resulted in minus 100 points (150 for older participants). All participants received $\$ 1$ for every 20,000 points they eamed.

Trials. Each trial began with the simultaneous presentation of a warning tone $(100 \mathrm{~Hz}$ for $50 \mathrm{~ms})$ and a central fixation cross. The Task 1 tone stimulus was then presented for $40 \mathrm{~ms}$, followed by the SOA and then the Task 2 digit stimulus. The fixation cross remained on the screen until it was replaced by the digit. The digit remained on the screen until either the participant made a right-hand keypress or $2,000 \mathrm{~ms}(3,000 \mathrm{~ms}$ for the older participants) elapsed. Meanwhile, responses to the tone were made with the left hand. The time between trials was $500 \mathrm{~ms}$.

Practice. There were two types of practice blocks: single task and dual task. Single-task practice blocks included 24 trials with only one type of task (tone, easy digit, or hard digit). Dual-task practice blocks included 
trials with a combination of the tone task and either the easy-digit task or the hard-digit task. Each dual-task practice block included only one SOA (either 150,500 , or $1,000 \mathrm{~ms}$ ). For all practice blocks, participants received feedback at the end of each trial, stating whether each response was correct, how long each response took, and the points accrued for that trial. All of Session 1 was considered practice.

Test blocks. All test blocks were dual-task blocks of 80 trials each. During test blocks, feedback after a trial was only given if the participant responded incorrectly. Also, at the end of a block (test or practice), participants received summary feedback. The summary gave average RT for each task, the number of correct and incorrect responses for each task, the total number of points for the block, and a cumulative point total for all previous blocks. During all blocks, the RT for each task was recorded, along with the accuracy of the response. If a response was incorrect, then it was coded according to type of error: wrong response, out of order (Task 2 response occurred first), too short (the participant anticipated the stimulus), or too long (there was no response during the time allowed for completion of the tasks following stimulus presentation).

Sessions. There were 3 sessions, with 13 blocks per session. Session 1 was devoted to practice of the tasks. Data collection occurred in Sessions 2 and 3. Session 1 began with 3 blocks of single-task practice, 1 for each of the tasks (tone, easy digit, hard digit). This was followed by a block of dual-task practice with the tone and the easy-digit tasks, using only the 1,000-Ins SOA. Then there was a similar dual-task block, using only the 150-ms SOA. Following this were the 2 final practice blocks, using all five SOAs, first with the easy-digit task, and then with the hard-digit task. There were then 6 regular dual-task blocks, 3 for the easy-digit task, and 3 for the hard-digit task.

Sessions 2 and 3 each started with 1 block of dual-task practice, using the tone task and the hard-digit task, and only the 500-ms SOA. Then there were 12 dual-task test blocks. The first 6 dual-task blocks included 3 blocks with the easy Task 2 and then 3 blocks with the hard Task 2 . These 6 blocks were then repeated. Therefore, Sessions 2 and 3 each contained 960 dual-task trials per participant.

Data analysis. Reaction times were analyzed only for trials where both Task 1 and Task 2 were correct. Practice blocks were not analyzed. Before analysis, outliers in the RT data were trimmed using a systematic algorithm (Schumacher et al., 1999). ${ }^{2}$ For the young participants, 1,102 trials ( $5 \%$ of the total correct trials) were removed by this trimming procedure. For the older participants, 1,072 trials ( $5 \%$ of the total correct trials) were removed.

Reaction times for Task 1 and Task 2 were analyzed separately, each with a 2 (age) $\times 2$ (Sessions 2 and 3) $\times 2$ (Task 2 difficulty) $\times 5$ (SOA) analysis of variance (ANOVA), with session, Task 2 difficulty, and SOA as repeated variables.

\section{Results}

\section{Reaction Times}

Figure 5 summarizes the mean Task 1 and Task 2 RTs for each age group on each session.

Task 1. The main effect of age on mean Task 1 RT was significant, $F(1,18)=28.05, p<.0001, \eta^{2}=.609$. The mean Task 1 RT was $517 \mathrm{~ms}$ for the older participants and $345 \mathrm{~ms}$ for the young participants. The main effect of session was significant, $F(1$, 18) $=29.0, p<.0001$. Task 1 RTs were $45 \mathrm{~ms}$ faster on Session 3. The main effect of SOA was small but significant, $F(4,72)=16.9$, $p<.0001$. This effect can be seen in Figure 5 as an increase in Task 1 RTs at the shortest SOA. The mean Task 1 RT when SOA $=50 \mathrm{~ms}$ was $23 \mathrm{~ms}$ longer than the mean RT when SOA $=1,000 \mathrm{~ms}$. However, the effect of Task 2 difficulty on Task 1 RTs .was not significant, $F(1,18)=.82$.

The main effect of SOA on Task 1 RTs was qualified by two interactions. The Age $\times$ SOA interaction was significant, $F(4$,
72) $=9.25, p<.0001, \eta^{2}=.346$. Inspection of Figure 5 reveals that the SOA effect on Task 1 RTs was only present for the older participants. Specifically, the difference between Task 1 RTs when $\mathrm{SOA}=50 \mathrm{~ms}$ and when $\mathrm{SOA}=1,000 \mathrm{~ms}$ was $5 \mathrm{~ms}$ for the young participants and $49 \mathrm{~ms}$ for the older participants. Finally, the Task 2 difficulty by SOA interaction for Task 1 RTs was significant, $F(4,72)=3.86, p=.01$. The effect of SOA on Task 1 RTs was larger when Task 2 was difficult. Although the Task 2 Difficulty $\times$ SOA interaction appears larger for the older participants, the Age $\times$ Task 2 Difficulty $\times$ SOA interaction was not significant, $F(4,72)=1.65, p=.172, \eta^{2}=.084$, observed power $=$ .482 . No other interactions were significant.

Task 2. All main effects on mean Task 2 RTs were significant. The older participants were slower than the younger participants, $F(1,18)=41.7, p<.0001, \eta^{2}=.699$. The main effect of session was significant, $F(1,18)=26.8, p<.0001$, as was the Age $\times$ Session interaction, $F(1,18)=4.88, p=.04, \eta^{2}=.213$. For the young participants, the mean Task $2 \mathrm{RT}$ was $27 \mathrm{~ms}$ shorter on Session 3 than on Session 2, and for the older participants, it was $69 \mathrm{~ms}$ shorter on Session 3. There was a main effect of Task 2 difficulty, $F(1,18)=77.1, p<.0001, \eta^{2}=.811$, and a main effect of SOA, $F(4,72)=198.8, p<.0001, \eta^{2}=.917$. Task 2 difficulty interacted with $\operatorname{SOA}, F(4,72)=11.9, p<.0001, \eta^{2}=$ 399. The effect of Task 2 difficulty on Task 2 RTs increased as SOA increased, yielding divergent PRP curves. The triple interaction of Age $\times$ Task 2 Difficulty $\times$ SOA was not significant, $F(4$, $72)=.846, p=.50, \eta^{2}=.045$, power $=.257$. This is consistent with the observation of diverging PRP curves in each age group. To further test the reliability of this pattern, separate ANOVAs were performed for each age group. For the young and older age groups, respectively, the Task 2 Difficulty $\times$ SOA interaction was significant, $F(4,36)=17.42, p<.0001, \eta^{2}=.659 ; F(4$, $36)=3.27, p=.022, \eta^{2}=.266$. The amount of divergence in the PRP curves can be quantified by subtracting the Task 2 difficulty effect at the shortest SOA from the Task 2 difficulty effect at the longest SOA. This yields a mean divergence measure of $44 \mathrm{~ms}$ for the young adults and $46 \mathrm{~ms}$ for the older adults.

However, for the Task 2 RTs, age did interact with SOA, $F(4$, $72)=34.5, p<.0001, \eta^{2}=.673$. The PRP effect (RT at SOA 50 minus RT at SOA 1,000), averaged over Task 2 difficulty, was larger for the older adults compared to the younger adults $(293 \mathrm{~ms}$ vs. $124 \mathrm{~ms}$ ).

Finally, the session effect on Task 2 RTs interacted with Task 2 difficulty, $F(1,18)=6.47, p=.02$, and with SOA, $F(4$, $72)=9.55, p<.0001$. The effect of Task 2 difficulty was $22 \mathrm{~ms}$ smaller in Session 3. The size of the PRP effect (RT at SOA 50 minus RT at SOA 1,000) was $45 \mathrm{~ms}$ smaller in Session 3. No other interactions were significant.

\footnotetext{
${ }^{2}$ With this procedure, the logarithm of each reaction time was obtained. Then, within each cell (participant by Task 2 difficulty by SOA), 10\% from each tail was trimmed. The standard deviation of the trimmed RT logarithms within each cell was determined. This standard deviation was multiplied by an expansion factor of 1.51 , to take into account the $10 \%$ tails that had initially been trimmed. The trimmed tails were then put back into the distribution. Values exceeding plus or minus 3.8955 expanded standard deviations were then discarded. Remaining RT logarithms were back transformed to the original RT scale for subsequent analyses.
} 

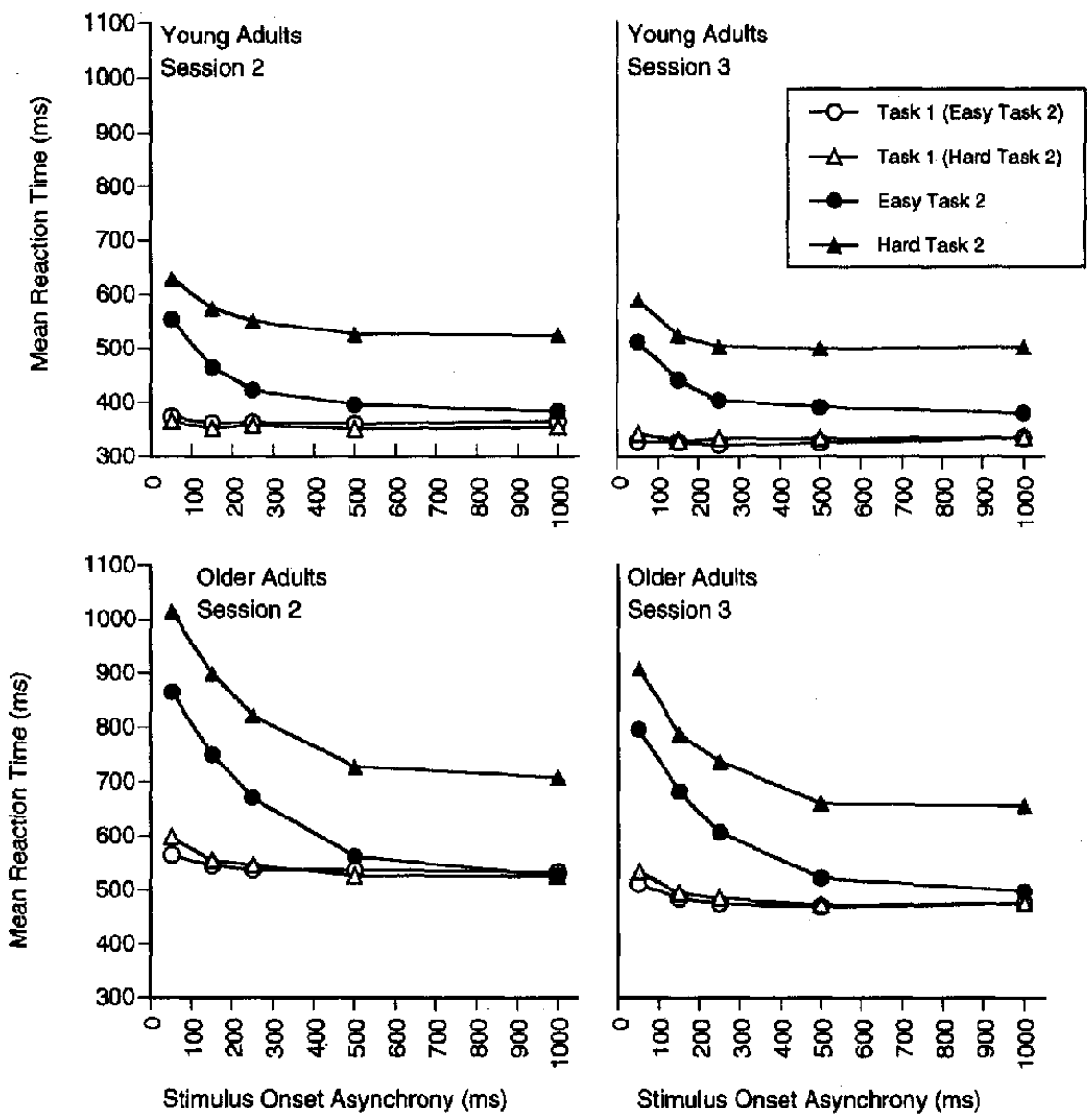

Figure 5. Mean reaction times (RTs) for Tasks 1 and 2 (T2) as a function of T2 response-selection difficulty and stimulus onset asynchrony (SOA) in Experiment 1. The top panels show mean RTs for the young adults, and the bottom panels show mean RTs for the older adults. The panels on the left depict mean RTs for Session 2, and the panels on the right depict mean RTs for Session 3. The T2 difficulty by SOA interaction for T2 RTs is significant in both age groups, evidenced by the diverging psychological refractory period curves. The data from the young adults are also published in Schumacher et al. (1999).

A common practice in some studies of cognitive aging has been to derive relative measures of dual-task performance (e.g., Somberg \& Salthouse, 1982). These relative measures take into account performance on the component tasks when they are performed singly. To enable comparisons with these previous studies, we calculated the proportion of the older adults' mean PRP effect relative to the young adults' mean PRP effect. The value of this ratio is 2.52 (PRP effect of older adults divided by PRP effect of young adults). We also calculated the proportion of the older adults' mean Task 1 RTs relative to the young adults' mean Task 1 RTs. The value of this ratio is 1.48 (Task $1 \mathrm{RT}$ of older adults divided by Task 1 RT of young adults). Mean Task 1 RTs were used because Task 1 is given priority by the PRP instructions and therefore provides an estimate of single-task RT.

From these two ratios, it can be seen that the normalized size of the older adults' mean PRP effect is much larger than would be expected based on their mean Task 1 RTs. At first blush, this outcome might be taken to suggest that the older adults are especially poor at dual-task performance, task scheduling, and executive control. However, as explained later, such an interpretation would be wrong. On the contrary, despite their relatively large PRP effects, the present older adults scheduled their tasks essentially in the same fashion as the young did, which is shown more fully later.

\section{Errors}

A 2 (age) $\times 2$ (session) ANOVA, with session as a withinparticipants variable, was used to analyze the error rates. The effect of age on error rates was marginally significant, $F(1$, $18)=4.35, p=.06$. Younger adults had more errors than did older adults, $6 \%$ versus $3 \%$, respectively. There were marginally fewer errors on Session $3(4 \%)$ than on Session $2(5 \%), F(1,18)=3.83$, $p=.066$. Of particular interest are the errors caused by out-oforder responses (where the participant responded to Task 2 before Task 1), which were infrequent for both age groups. Young adults made two $(.02 \%)$ out-of-order responses during Session 3 . Older adults made three $(.03 \%)$ out-of-order responses during Session 3 . 


\section{Summary}

The results of Experiment 1 show that both young and older adults had divergent PRP curves, thus suggesting that both age groups used a similar task-coordination strategy that allowed some overlap between the response-selection stages of both tasks. There was no evidence for a deficit in executive control of the processing of each task, because out-of-order errors were rare, especially by the third session. However, the older adults also had a larger PRP effect than did the young adults ( $293 \mathrm{~ms}$ vs. $124 \mathrm{~ms}$ ). To identify possible sources of these similarities and differences, we quantified the results of Experiment 1, using the EPIC architecture and a particular type of model specified within it.

\section{The Strategic Response-Deferment Model}

The particular type of model that we used here to interpret the results of Experiment 1 is called the strategic response-deferment (SRD) model, a special case of the AEC models described earlier (Figure 3; Meyer et al., 1995; Meyer \& Kieras, 1997a, 1997b). This model has been successful in accounting for the results of several experiments where the PRP curves diverge. In the SRD model, the Task 2 lockout point is set to occur after the Task 2 response-selection stage. This enables response selection to proceed in parallel for both Task 1 and Task 2, and it is consistent with the observation of diverging PRP curves. Because we found that the PRP curves of both older and younger adults diverged in Experiment 1, the SRD model is appropriate for modeling the data from both of these groups.

Specifically, we address two questions: (a) Are the taskcoordination strategies used by young and older adults the same? and (b) Can the pattern of PRP data observed for the older adults be explained with a single, generalized slowing factor (i.e., an increase of the cognitive-processor cycle duration)? To answer these questions, we constructed four separate SRD models. The first of these provided the best fit for the young adults' PRP data. The second SRD model provided the best fit for the older adults' PRP. By comparing the parameters of these two models, we may see where age differences in dual-task performance are localized. The third and fourth SRD models were constructed to test and reject the prediction that generalized slowing alone can explain the older adults' PRP data.

Our approach involved applying theoretical RT equations (Meyer \& Kieras, 1997a) whose component variables are based on parameters of the SRD model (see Table 2). With these equations and parameters, the theoretical mean RTs and parameter values based on this model can be estimated for both young and older adults as a function of SOA. We obtained such estimates in the cases of mean Task 1 RT and mean Task 2 RTs at the shortest and longest SOAs ( $50 \mathrm{~ms}$ and $1,000 \mathrm{~ms}$ ), for each level of Task 2 difficulty. This captured the size of the PRP effect as well as the interaction of SOA and Task 2 difficulty effects on Task 2 RTs. The theoretical parameters and equations associated with the SRD model are explained in more detail in the Appendix.

Figure 6 shows the empirical mean RTs versus the theoretical mean RTs from the SRD model for young and older adults. These values come from the SRD model that affords the best fit to the observed PRP data for each age group. ${ }^{3}$ The parameter values for each model are shown in Table 3. The root-mean-squared error
Table 2

Parameters of the Strategic Response-Deferment and Other Adaptive Executive-Control Models for the Psychological Refractory Period Procedure

\begin{tabular}{|c|c|c|}
\hline System component and parameter name & Symbol & Type \\
\hline \multicolumn{3}{|l|}{ Cognitive processor } \\
\hline Cycle duration & $t_{c}$ & $\mathbf{P}$ \\
\hline Working-memory gating time & $t_{\mathrm{g}}$ & $\mathbf{P}$ \\
\hline \multicolumn{3}{|l|}{ Perceptual processors } \\
\hline Stimulus identification time & $t_{i}$ & $\mathrm{D}$ \\
\hline \multicolumn{3}{|l|}{ Motor processors } \\
\hline No. of movement features & $\mathrm{n}_{\mathrm{f}}$ & $\mathbf{P}$ \\
\hline Preparation time per feature & $t_{f}$ & $\mathrm{P}$ \\
\hline Movement production time & $t_{m}$ & $\mathbf{P}$ \\
\hline \multicolumn{3}{|l|}{ Task processes } \\
\hline No. of selection cycles & $\mathbf{n}_{\mathbf{s}}$ & $\mathrm{P}, \mathrm{D}$ \\
\hline Response-selection time & $t_{s}$ & P, D \\
\hline \multicolumn{3}{|l|}{ Executive process } \\
\hline Unlocking-onset latency & $\mathbf{t u}_{\mathbf{u}}$ & D \\
\hline Minimum unlocking duration & $t_{y}$ & P, D \\
\hline \multicolumn{3}{|l|}{ Apparatus } \\
\hline Response-transduction time & $t_{T}$ & $\mathbf{P}$ \\
\hline
\end{tabular}

Note. For the sources of the parameter's value, $\mathrm{P}$ is predetermined and $\mathrm{D}$ is derived from the data.

(RMSE) between the empirical theoretical RTs for the young adults is $12 \mathrm{~ms}$ and for the older adults is $7 \mathrm{~ms}$. The good fits suggest that both young and older adults may overlap response selection for each task and that each age group was using a late Task 2 lockout point.

There are three main differences between the model parameters that produced the theoretical mean RTs for the young and older adults shown in Figure 6 . First, the cognitive-processor cycle duration is longer for the older adults. In the SRD model, we set the older adults' cycle duration to $56 \mathrm{~ms}$. A second main difference between the SRD models for the young and older adults is that the estimated perceptual identification times are longer for the older adults, especially in Task 1 (the tone discrimination task). Third, the model for the older adults takes longer to complete the Task 2 unlocking process. Altogether, these differences account for the larger PRP effects that we observed in the data from the older adults.

In contrast to the preceding SRD model for the older adults, we also tested whether their performance can be characterized solely in terms of generalized slowing. This was done by assessing two other SRD models for the older adults' PRP data. In each of these alternative models, it was assumed that the only difference between the age groups was in cognitive-processor cycle duration. The parameter values for each of these models are shown in Table 4. The theoretical RTs from these models, along with the empirical mean RTs, are shown in Figure 7.

The cycle duration used in the first generalized-slowing SRD model was $56 \mathrm{~ms}$. It can be seen that all of the empirical mean RTs are greatly underestimated by this model (RMSE $=163 \mathrm{~ms}$ ). This finding further establishes the reality of the strategy differences

\footnotetext{
${ }^{3}$ The models were fit to the group means, not to individual participants'
} 

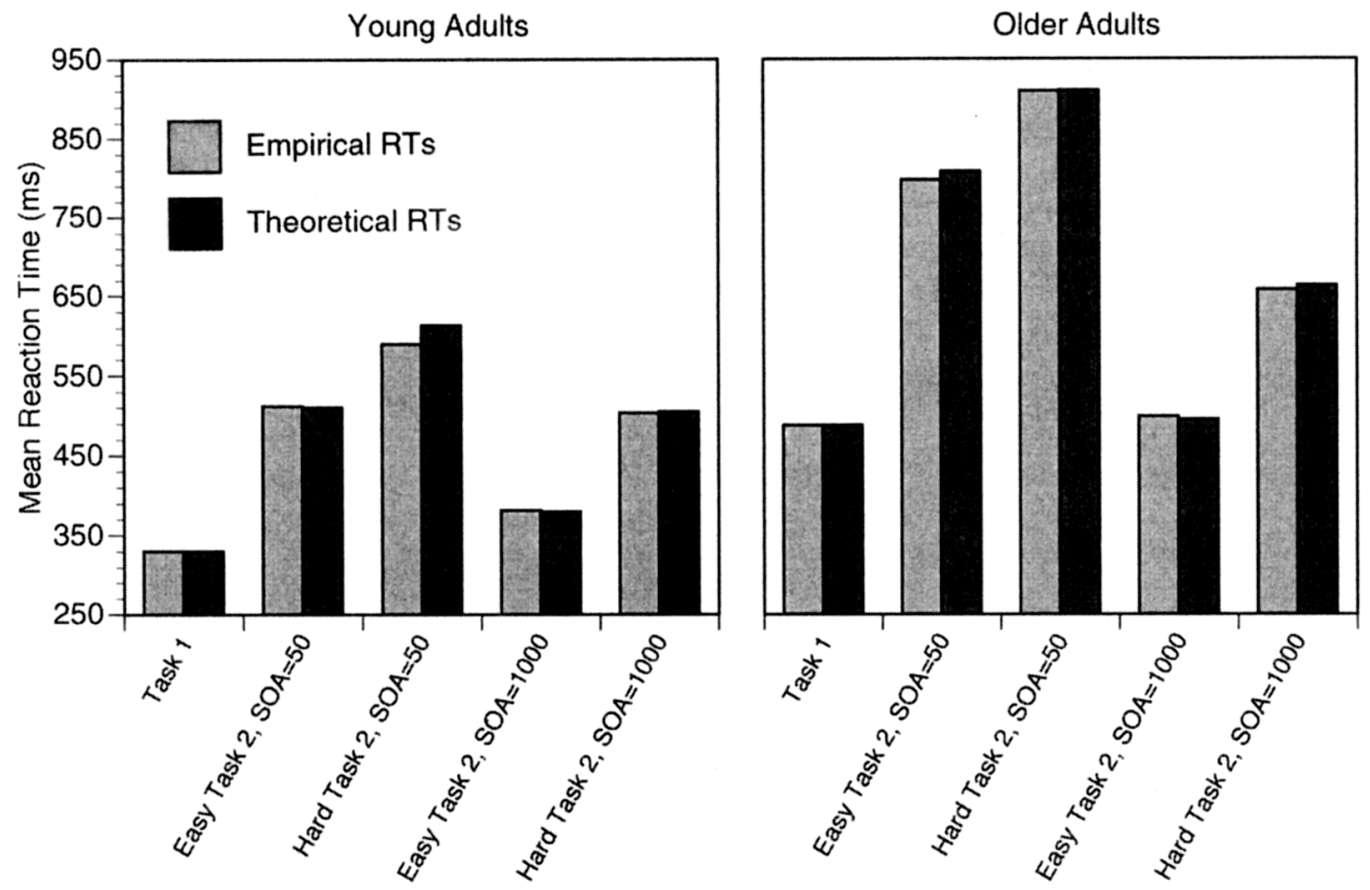

Figure 6. Empirical and theoretical mean reaction times (RTs) from the best fitting strategic responsedeferment models for Experiment 1. The panel on the left depicts RTs for the young adults (root-mean-square error $[R M S E]=11.60)$. The panel on the right depicts RTs for the older adults (RMSE $=6.6$ ). SOA = stimulus onset asynchrony.

that were incorporated in the prior, better fitting SRD models (cf. Figure 6). However, it might be argued that a $12 \%$ increase in the cognitive-processor cycle duration $(50 \mathrm{~ms}$ to $56 \mathrm{~ms})$ underestimated generalized slowing and is not long enough. For example, in studies where the RTs of older adults are plotted against the RTs of young adults, the resulting regression equations usually have a slope of 1.5-2.0 (Hartley, 1992). Perhaps the cognitive-processor cycle duration in the SRD model of older adults' RTs should be 1.5 times the cognitive-processor cycle duration in the model of young adults' RTs.

Therefore, in the second generalized-slowing SRD model, the cycle duration was set at $75 \mathrm{~ms}$. The theoretical RTs from this model are also shown in Figure 7. A better fit was obtained for Task $1 \mathrm{RT}$ and for Task 2 RTs at the short SOA than in our previous-generalized slowing SRD model. However, the theoretical RTs for Task 2 at the long SOA are now too long, and the RMSE is substantially larger ( $54 \mathrm{~ms}$ ) than in the original best fitting SRD model (cf. Figure 6). In fact, simply increasing the cognitive-processor cycle duration cannot capture the pattern of the older adults' PRP data. Again, this demonstrates the importance of not only generalized slowing but also task-coordination strategy differences in characterizing the PRP performance of young and older adults.

\section{Discussion}

In Experiment 1, both young and older adults had divergent PRP curves. This suggests that the task-coordination strategy for each age group involved a late Task 2 lockout point that allowed the
Task 1 and Task 2 response-selection stages to overlap. Also, the older adults were slower overall and had a larger PRP effect than did the young adults.

Three sources for the larger PRP effect were identified through analysis based on the SRD models. First, cognitive processing slows with age. This was accommodated in the SRD model with a longer cognitive-processor cycle duration for the older adults. In this way, our results are consistent with the generalized-slowing hypothesis (Bashore, 1994; Cerella, 1985; Salthouse, 1985, 1996). However, slowed cognitive processing alone is not enough to explain the present data, because two SRD models in which only the cognitive-processor cycle duration increased with age failed to provide satisfactory estimates of the RTs. In addition to the longer cognitive-processor cycle duration, the perceptual identification times estimated through the SRD model were longer for the older adults than expected from the increased cognitive-processor cycle duration. Thus, we have evidence of process-specific slowing, which is modality dependent, in addition to generalized slowing. A third source of the older adults' larger PRP effect involves their task-coordination strategy. Both young and older adults used a similar, late (e.g., after response selection) Task 2 lockout point. However, the older adults were more cautious in terms of their chosen Task 1 unlocking event. They waited longer than the young adults did before resuming Task 2 processing, and this extra strategic delay contributed to the size of the older adults' PRP effect.

The results of Experiment 1 and the SRD models also demonstrate that the older adults' executive control over the two tasks is 
Table 3

Parameter Values for Best Fitting Strategic Response-Deferment Models as a Function of Age Group and Task Type

\begin{tabular}{|c|c|c|c|c|c|c|}
\hline \multirow[b]{2}{*}{ Parameter name and symbol } & \multicolumn{3}{|c|}{ Young adults } & \multicolumn{3}{|c|}{ Older adults } \\
\hline & $\mathrm{T} 1$ & $\mathrm{~T} 2 \mathrm{E}$ & $\mathrm{T} 2 \mathrm{H}$ & $\mathrm{T} 1$ & T2E & $\mathrm{T} 2 \mathrm{H}$ \\
\hline \multicolumn{7}{|l|}{ Cycle duration } \\
\hline $\begin{array}{l}t_{c} \\
\text { Working-memory gating time }\end{array}$ & 50 & 50 & 50 & 56 & 56 & 56 \\
\hline Stimulus identification time $_{{ }_{\mathrm{g}}}^{\mathrm{t}_{\mathrm{g}}}$ & 25 & 25 & 25 & 28 & 28 & 28 \\
\hline No. of movement features & 120 & 121 & 121 & 254 & 205 & 205 \\
\hline $\begin{array}{l}\mathbf{n}_{\mathrm{f}} \\
\text { Movement production time }\end{array}$ & 1 & 2 & 2 & 1 & 2 & 2 \\
\hline $\begin{array}{l}t_{\text {mn }} \\
\text { No. of selection cycles }\end{array}$ & 100 & 150 & 150 & 112 & 168 & 168 \\
\hline $\begin{array}{l}\mathrm{n}_{\mathrm{s}} \\
\text { Response-selection time }\end{array}$ & 1.5 & 1.5 & 4 & 1.5 & 1.5 & 4.5 \\
\hline $\begin{array}{c}t_{s} \\
\text { Unlocking-onset latency }\end{array}$ & 75 & 75 & 200 & 84 & 84 & 252 \\
\hline $\begin{array}{l}t_{\mathrm{u}} \\
\text { Minimum unlocking duration }\end{array}$ & 100 & 100 & 100 & 90 & 90 & 90 \\
\hline $\begin{array}{l}\mathrm{t}_{\mathrm{v}} \\
\text { Response-transduction time }\end{array}$ & 80 & 80 & 80 & 224 & 224 & 224 \\
\hline $\mathrm{t}_{\mathrm{T}}$ & 10 & 10 & 10 & 10 & 10 & 10 \\
\hline
\end{tabular}

Note. $\mathrm{T} 1=$ Task $1 ; \mathrm{T} 2 \mathrm{E}=$ easy Task $2 ; \mathrm{T} 2 \mathrm{H}=$ hard Task 2.

impressively intact. For example, the older adults produced relatively flat Task 1 RTs, showing that they were able to obey the PRP instructions to give Task 1 priority. Although their SOA effect is significant for Task 1 , the size of the effect is small compared to the size of the SOA effect on Task 2 . In addition, out-of-order responses were rare for both age groups, again indicating that the older adults were able to exert effective executive control.

To further examine the role of strategy differences in age effects on dual-task performance, we conducted a second experiment that involved a dual-task situation in which young adults typically use a more cautious strategy than they did for Experiment 1 (Meyer et al., 1995). Instead of a late Task 2 lockout point (e.g., after Task 2 response selection), a cautious strategy has an early Task 2 lockout point (e.g., before Task 2 response selection). This is cautious because it allows less overlap of the processing stages for each task. In the PRP procedure, this strategy produces additive effects of Task 2 response-selection difficulty and SOA on mean Task 2 RTs (parallel PRP curves). The fact that different task demands lead to different patterns in the PRP data indicates that people are flexible in their dual-task coordination (in contrast to rigid bottleneck models). Experiment 1 suggested that both young and older adults are able to overlap the response-selection stages for two tasks. However, these results do not tell us whether older adults can use a different task-coordination strategy. Perhaps the strategy used by the older adults in Experiment 1 is the only one that they can use. Therefore, we tested young and older adults with a PRP procedure that leads young adults to adopt an early Task 2 lockout point. We were especially interested in whether older adults would also use an early Task 2 lockout point and whether any other aspect of their task-coordination strategy would differ from the young adults.

\section{Experiment 2}

Experiment 2 was similar to Experiment 1 except that we made Task 1 more difficult: It included four tones instead of two. The easy and hard versions of Task 2 were identical to those used in Experiment 1, as were the SOAs. Previous research in our lab has shown that increasing the difficulty of Task 1 induces a cautious task-coordination strategy in young adults (Meyer et al., 1995). The question of present interest then becomes, how do older adults perform under such conditions?

\section{Method}

\section{Participants}

Ten new right-handed older participants ( 5 male and 5 female), aged 60-69 years with a mean age of 66.2 years, were recruited from the University of Michigan Geriatrics Center human participants core. No participant had neurological (e.g., strokes or seizures) or psychiatric (e.g., depression) disorders. This was assessed from information provided by the Geriatrics Center and from the initial phone contact with the participants. As in Experiment 1, the level of participants' education was high: One participant had some college, 2 held bachelor's degrees, and 7 had a master's degree or higher. Participants reported themselves as having either good, very good, or excellent health. The modal responses were good and very good. There were no responses less than good. The participants had normal or corrected-to-normal vision. Participants who required hearing aids were excluded from the study. Participants were paid an hourly rate, plus bonuses based on good performance.

Nine new right-handed young adult participants ( 5 male and 4 female), aged 20-23 years with a mean age of 21.4 years, were recruited from the University of Michigan campus. They had normal or corrected-to-normal vision, and they were paid an hourly rate plus bonuses based on good performance. 
Table 4

Parameter Values for Two Generalized-Slowing Strategic Response-Deferment Models as a Function of Task Type

\begin{tabular}{|c|c|c|c|c|c|c|}
\hline \multirow[b]{2}{*}{ Parameter name and symbol } & \multicolumn{3}{|c|}{$12 \%$ slowing } & \multicolumn{3}{|c|}{$50 \%$ slowing } \\
\hline & $\mathbf{T} 1$ & $\mathrm{~T} 2 \mathrm{E}$ & $\mathrm{T} 2 \mathrm{H}$ & $\mathrm{T} 1$ & $\mathrm{~T} 2 \mathrm{E}$ & $\mathrm{T} 2 \mathrm{H}$ \\
\hline \multicolumn{7}{|l|}{ Cycle duration } \\
\hline $\begin{array}{l}t_{c} \\
\text { Working-memory gating time }\end{array}$ & 56 & 56 & 56 & 75 & 75 & 75 \\
\hline Stimulus identification time & 28 & 28 & 28 & 38 & 38 & 38 \\
\hline $\begin{array}{l}{ }_{i} \\
\text { No. of movement features }\end{array}$ & 134 & 134 & 134 & 254 & 205 & 205 \\
\hline $\begin{array}{l}n_{f} \\
\text { Movement production time }\end{array}$ & 1 & 2 & 2 & 1 & 2 & 2 \\
\hline $\begin{array}{l}t_{m} \\
\text { No. of selection cycles }\end{array}$ & 112 & 168 & 168 & 150 & 225 & 225 \\
\hline $\begin{array}{l}\mathrm{n}_{\mathrm{s}} \\
\text { Response-selection time }\end{array}$ & 1.5 & 1.5 & 4 & 1.5 & 1.5 & 4.5 \\
\hline $\begin{array}{l}\mathrm{t}_{\mathrm{s}} \\
\text { Unlocking-onset latency }\end{array}$ & 84 & 84 & 224 & 113 & 113 & 300 \\
\hline $\begin{array}{l}t_{u} \\
\text { Minimum unlocking duration }\end{array}$ & 112 & 112 & 112 & 150 & 150 & 150 \\
\hline $\begin{array}{l}t_{v} \\
\text { Response-transduction time }\end{array}$ & 90 & 90 & 90 & 120 & 120 & 120 \\
\hline $\mathrm{t}_{\mathrm{r}}$ & 10 & 10 & 10 & 10 & 10 & 10 \\
\hline
\end{tabular}

Note. $\mathrm{T} 1=$ Task $1 ; \mathrm{T} 2 \mathrm{E}=$ easy Task $2 ; \mathrm{T} 2 \mathrm{H}=$ hard Task 2.

\section{Apparatus}

The apparatus was the same as in Experiment 1.

\section{Stimuli and Responses}

Participants made responses to two stimuli (a tone and a digit) on each trial. The Task 1 tones were either $333 \mathrm{~Hz}, 500 \mathrm{~Hz}, 1,120 \mathrm{~Hz}$, or $1,450 \mathrm{~Hz}$, and the responses for them were keypresses by the left little finger, left ring finger, left middle finger, or the left index finger, respectively. There were easy and hard versions of the visual-manual Task 2, identical to Task 2 in Experiment 1.

The Task 1 tone was always presented before the Task 2 digit, and participants had to respond to the tone first. Stimulus onset asynchrony, the time between the onsets of the tone and the digit, varied from trial to trial within a block. The five SOAs were the same as in Experiment $1(50,150$, 250,500 , or $1,000 \mathrm{~ms}$ ). The particular SOA, tone, and digit for each trial were selected randomly from a set of SOA, tone, and digit combinations predetermined for each block.

\section{Procedure}

The SOA and task combinations were counterbalanced to ensure that each possible combination was used an equal number of times. There were three sessions for each participant on separate days within 1 week. Each session lasted about $1.5 \mathrm{hr}$.

Participants received written instructions about the tasks and the payoff structure. The instructions stated that the response to the auditory task (Task 1) must come before the response to the visual task (Task 2). After the participant read the instructions, the experimenter described the tasks to make sure that the participant understood the instructions.

Points. The point system was identical to that of Experiment 1.

Trials. Each trial began with the simultaneous presentation of a warning tone $(880 \mathrm{~Hz}$ for $50 \mathrm{~ms})$ and a central fixation cross. A Task 1 tone was then presented for $40 \mathrm{~ms}$, followed by the SOA and then a Task 2 digit. The fixation cross remained on the screen until it was replaced by the digit. The digit remained on the screen until either the participant made a right-hand keypress or $2,000 \mathrm{~ms}(3,000 \mathrm{~ms}$ for the older adults) elapsed. The time between trials was $500 \mathrm{~ms}$.

Practice. There were two types of practice blocks, single task and dual task. Single-task practice blocks included 24 trials of one task (tone, easy digit, or hard digit). Dual-task practice blocks included trials with a combination of the tone task and either the easy- or hard-digit task. The dual-task practice blocks used only one of the five SOAs $(500 \mathrm{~ms})$. For all practice blocks, participants received feedback at the end of each trial, stating whether each response was correct, how long each response took, and the points accrued for that trial.

Test blocks. The test blocks were the same as in Experiment 1, except that there were 48 trials per block.

Sessions. There were three sessions, with 18 blocks per session. Session 1 was devoted to practice of the tasks. Data collection occurred in Sessions 2 and 3. Session 1 began with six blocks ( 32 trials each) of single-task practice, two for each of the tasks (tone, easy digit, hard digit). This was followed by two blocks ( 32 trials each) of dual-task practice with the tone and the easy-digit tasks, using only the $500-\mathrm{ms}$ SOA. The first dual-task practice block used full feedback (the participant received information about the correctness of each response and the points for each task on every trial). The second dual-task practice block used partial feedback (the participant received feedback only when there was an error on one or both tasks). Two dual-task practice blocks ( 32 trials each, 500-ms SOA, first block full feedback, second block partial feedback) with the hard-digit task followed. Then came eight dual-task blocks, four for the easy-digit task and four for the hard-digit task. In these blocks, all five SOAs were included, and participants received feedback after a trial only if an error occurred. Session 1 was identical for all participants.

Sessions 2 and 3 each started with two blocks of dual-task practice (one easy-digit Task 2, one hard-digit Task 2), using only the 500-ms SOA. Then there were four blocks of dual-task trials with one of the Task 2 mappings followed by four blocks of dual-task trials with the other mapping. These eight blocks were then repeated. Thus, Sessions 2 and 3 each contained 768 dual-task trials. The type of first test block (easy-digit task 


\section{Empirical RTs}

Theoretical RTs, $12 \%$ Slowing

Theoretical RTs, $50 \%$ Slowing

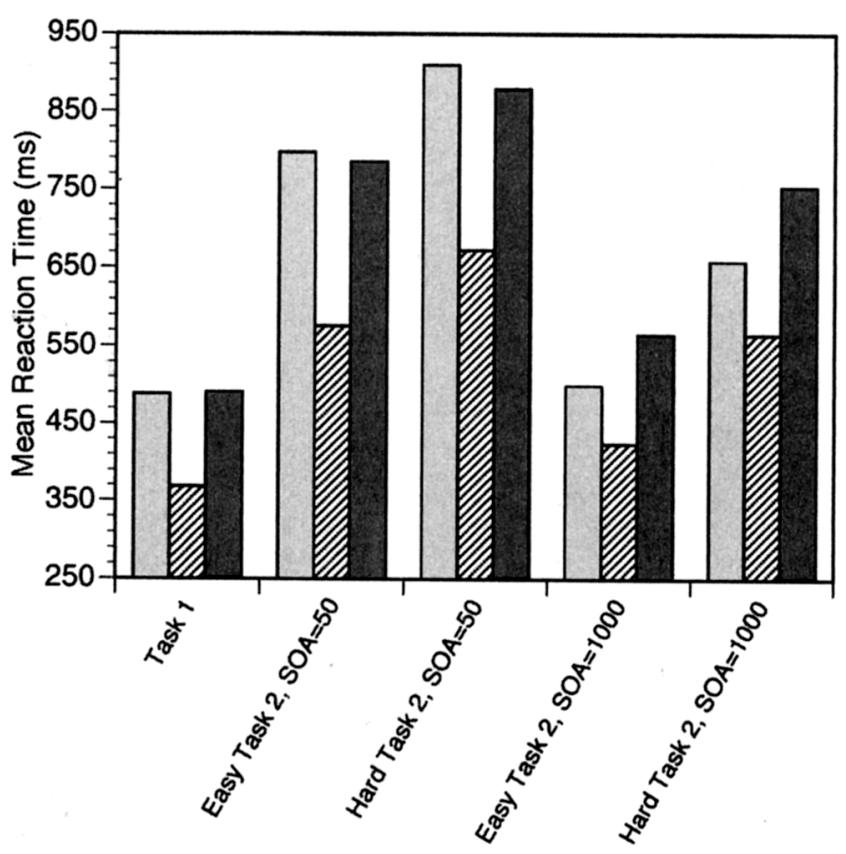

Figure 7. Empirical and theoretical mean reaction times (RTs) from two additional strategic response-deferment models that tested the generalizedslowing hypothesis for the older adults in Experiment 1. When the cognitive-processor cycle duration was $56 \mathrm{~ms}$ ( $12 \%$ slowing), root-meansquare error $(\mathrm{RMSE})=163.4$. When the cognitive-processor cycle duration was $75 \mathrm{~ms}$ (50\% slowing), RMSE $=54.0$. Neither of these models provides as good a fit as the one whose theoretical RTs are depicted in Figure 6. E T2 $=$ easy task $2 ; \mathrm{H} \mathrm{T2}=$ hard task $2 ; \mathrm{SOA}=$ stimulus onset asynchrony.

or hard-digit task) was counterbalanced across participants. Sessions 2 and 3 were identical for each participant.

Data analysis. Reaction time was analyzed only for trials on which both Task 1 and Task 2 responses were correct. Practice blocks were not analyzed. Before analysis, the RT data were trimmed with the same procedure as in Experiment 1. For the young participants, 507 trials ( $4 \%$ of the total correct trials) were removed by this procedure. For the older participants, 454 trials ( $3 \%$ of the total correct trials) were removed.

Reaction times for Task 1 and Task 2 were analyzed separately through 2 (age) $\times 2$ (session) $\times 2$ (Task 2 difficulty) $\times 5$ (SOA) ANOVAs, with session, Task 2 difficulty, and SOA as repeated variables.

\section{Results}

\section{Reaction Times}

Figure 8 summarizes the Task 1 and Task 2 RTs for each age group in Sessions 2 and 3.

Task 1. All main effects on mean Task 1 RTs were significant.
The mean Task 1 RT was $869 \mathrm{~ms}$ for the older adults and $553 \mathrm{~ms}$ for the young adults, $F(1,17)=40.1, p<.0001, \eta^{2}=.703$. The main effect of session was significant, $F(1,17)=27.6, p<.0001$, $\eta^{2}=.619$. Mean Task 1 RTs were $50 \mathrm{~ms}$ shorter in Session 3 than in Session 2. The effect of Task 2 difficulty on Task 1 RTs was significant, $F(1,17)=22.0, p<.0001, \eta^{2}=.564$. RTs for Task 1 were faster when Task 2 was easy. Although this effect was larger for the older adults, the Task 2 Difficulty $\times$ Age interaction was only marginally reliable, $F(1,17)=3.87, p=.066, \eta^{2}=.186$, power $=.459$. The main effect of SOA was significant, $F(4$, $68)=16.3, p<.0001, \eta^{2}=.484$; Task 1 RTs increased at the shortest SOA. There was also a significant Task 2 Difficulty $\times$ SOA interaction, $F(4,68)=4.96, p=.0014, \eta^{2}=.215$. The effect of SOA on Task 1 RTs was larger when Task 2 was hard. Unlike in Experiment 1, the Age $\times$ SOA interaction in the Task 1 RTs was not significant, $F(4,68)=1.02, p=.404, \eta^{2}=.056$, power $=.305$. No other interactions were significant for Task 1 .

Task 2. All main effects on mean Task 2 RTs were significant. Older adults were slower than young adults for all combinations of Task 2 difficulty and SOA, $F(1,17)=78.6, p<.0001, \eta^{2}=.822$. Participants were on average $68 \mathrm{~ms}$ faster at Task 2 in Session 3 than in Session 2, $F(1,17)=50.7, p<.0001, \eta^{2}=.744$. This effect of session was modulated by two interactions. First, there was a significant Session $\times$ Task 2 Difficulty interaction in mean Task 2 RTs, $F(1,17)=8.42, p=.011, \eta^{2}=.325$. There was more benefit from practice when Task 2 was difficult. Reaction times for the hard Task 2 were $90 \mathrm{~ms}$ faster in Session 3, whereas the easy Task 2 RTs were $49 \mathrm{~ms}$ faster in Session 3. There was also a significant Session $\times$ SOA interaction, $F(4,68)=6.18, p<$ $.0001, \eta^{2}=.267$. For mean Task 2 RTs, the benefit of practice was greatest at the shortest SOA.

The effect of Task 2 difficulty on mean Task 2 RTs was significant, $F(1,17)=48.9, p<.0001, \eta^{2}=.735$. At all SOAs, the hard Task 2 was performed more slowly than the easy Task 2 . The Task 2 difficulty effect was larger for the older adults, but this interaction was only marginally reliable, $F(1,17)=4.32, p<$ $.053, \eta^{2}=.203$, power $=.500$. The effect of SOA was significant, $F(4,68)=358.4, p<.0001, n^{2}=.953$. Task 2 RTs decreased as SOA increased. SOA also interacted with age, $F(4,68)=30.5$, $p<.0001, \eta^{2}=.643$. Older adults had larger PRP effects than young adults (662 ms vs. $369 \mathrm{~ms}$ ).

Finally, it is important to note the lack of a Task 2 Difficulty $X$ SOA interaction in the mean Task $2 \mathrm{RTs}, F(4,68)=1.11, p=.36$, $\eta^{2}=.061$, power $=.33$. The PRP curves are essentially parallel (vertically equidistant), and this is true for both age groups. As in Experiment 1, we calculated the amount of divergence for each participant (effect of Task 2 difficulty at the longest SOA minus the effect of Task 2 difficulty at the shortest SOA). For the young adults, the mean divergence score was $-5 \mathrm{~ms}$, and for the older adults the mean divergence score was -19 ms [mean difference $=13.44 \mathrm{~ms}, \mathrm{t}(17)=.530, p=.603, S E$ difference $=25]$. Consistent with this, the Task 2 Difficulty $\times$ SOA $\times$ Age interaction in mean Task 2 RTs was not significant, $F(4,68)=0.86$, $p=.494, \eta^{2}=.048$, power $=.259$.

As with Experiment 1, we also calculated some relative measures of dual-task performance. The ratio of the older adults' mean PRP effect relative to the young adults' mean PRP effect was 1.85 . The ratio of the older adults' mean Task 1 RT relative to the young adults' mean Task $1 \mathrm{RT}$ was 1.59. Thus, in both Experiments 1 

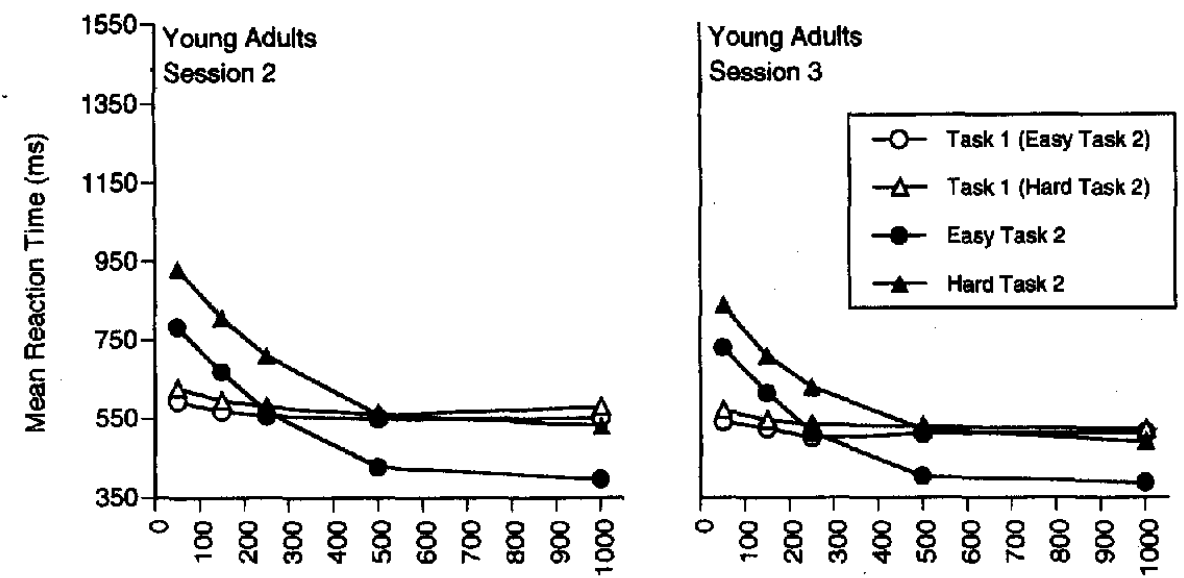

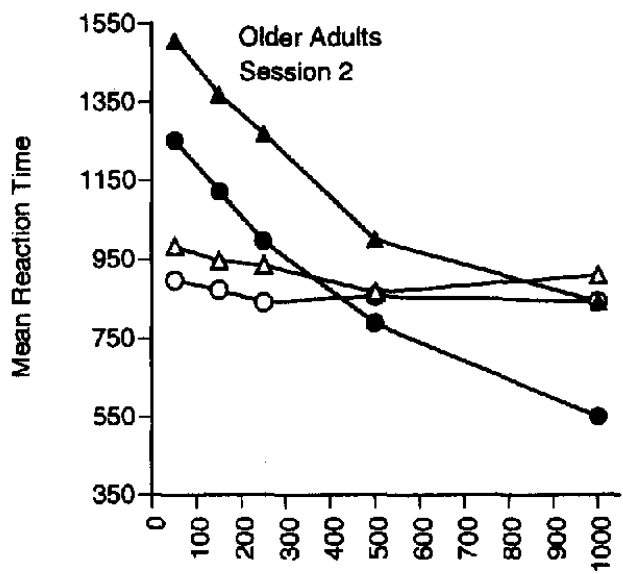

Stimulus Onset Asynchrony (ms)

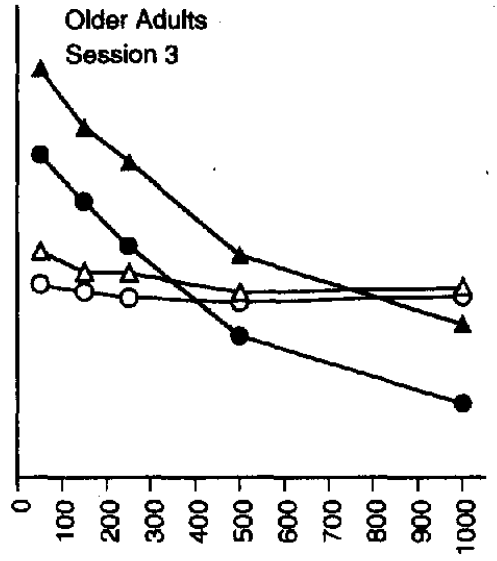

Stimulus Onset Asynchrony (ms)

Figure 8. Mean reaction times (RTs) for Tasks 1 and 2 (T2) as a function of $\mathrm{T} 2$ response-selection difficulty and stimulus onset asynchrony (SOA) in Experiment 2. The top panels show mean RTs for the young adults, and the bottom panels show mean RTs for the older adults. The panels on the left depict mean RTs for Session 2, and the panels on the right depict mean RTs for Session 3. There was no interaction of T2 difficulty and SOA for T2 RTs in either age group, evidenced by the equidistant psychology refractory period curves.

and 2, the PRP effect was larger in the older adults than expected from the age-related slowing for Task 1 . However, this disproportionate effect of aging was more pronounced in Experiment 1 than in Experiment 2 (in Experiment 1, the older adults' PRP effect was 2.52 times larger than the young adults' PRP effect, and the mean RT on Task 1 for older adults was 1.48 times larger than the mean RT for young adults).

\section{Errors}

The number of trials that contained errors was analyzed with a 2 (age) $\times 2$ (session) ANOVA. In contrast to the results of Experiment 1, the main effect of age was not significant, $F(1,17)=$ 863. Participants had on average $7 \%$ (6\% for young adults, $7 \%$ for older adults) trials with errors per session. The main effect of session was significant, $F(1,17)=5.22, p=.0354$, with fewer errors in Session 3 than in Session 2 (6\% and $7 \%$, respectively). The Age $\times$ Session interaction was marginally reliable, $F(1$,
$17)=3.887, p=.065$. There were very few out-of-order responses by either age group, $0.65 \%$ for young adults and $0.46 \%$ for older adults.

\section{Summary}

The results of Experiment 2 show that both young and older adults had parallel PRP curves, thus indicating that both age groups used a similar strategy that precluded overlap between the response-selection stages of both tasks. There was no evidence for a deficit in executive control of the processing of each task, because out-of-order errors were rare. However, the older adults had a larger PRP effect than did the young adults (293 ms vs. 124 $\mathrm{ms})$. To identify possible sources of these similarities and differences, we interpreted the results of Experiment 2, using the EPIC architecture and an AEC model different from the previous SRD model used for Experiment 1. 


\section{The Response-Selection Lockout Model}

Because the parallel PRP curves in Experiment 2 embody additive effects of SOA and Task 2 difficulty, the AEC model that best characterizes them is a response-selection lockout (RSL) model rather than an SRD model (cf. Experiment 1). Under the RSL model, the Task 2 lockout point is set to occur before the Task 2 response-selection stage instead of after it. As a result, Task 2 processing pauses after the Task 2 stimulus is identified, but before the Task 2 response is selected, yielding additive PRP curves. This use of a relatively early (preselection) lockout point may have occurred in Experiment 2 to help preclude out-of-order Task 2 responses in the context of a difficult primary Task 1 (Meyer \& Kieras, 1997b, 1999).

The RSL model involves some of the same parameters that were specified for the prior SRD model (Table 2). Several RT equations are also associated with the RSL model, and these equations may be used to account for the mean Task 1 RT and the mean Task 2 RTs at the shortest and longest SOAs $(50 \mathrm{~ms}$ and $1,000 \mathrm{~ms}$, respectively), for each age group. As in Experiment 1, these equations capture the sizes of the PRP effects together with the amount of interaction between SOA and Task 2 difficulty. Further details about the RT equations for the RSL model are contained in the Appendix.

Specifically, we were interested in two questions with respect to the results of Experiment 2: (a) Can the pattern of PRP data observed for the older adults be explained by a single, generalized slowing factor (i.e., a longer cognitive-processor cycle duration)? and (b) Are the task-coordination strategies used by young and older adults the same or different? To answer these questions, we constructed an RSL model that provided the best fit for the young adult PRP data. We then used this model as a starting point to account for the older adult PRP data, thus giving us insight about the similarities and differences between these age groups.

Figure 9 shows the empirical mean RTs versus the theoretical mean RTs for the young and older adults. These values come from the RSL model that affords the best fit to the observed PRP data in each age group on the basis of parameter values shown in Table 5. The RMSE of the theoretical versus empirical Task 2 RTs was $4 \mathrm{~ms}$ for the young adults and $11 \mathrm{~ms}$ for the older adults.

Like the findings from the SRD models of Experiment 1, there were three main differences between the parameter values used for modeling the PRP data of the young and old adults in Experiment 2 . First, the cognitive-processor cycle duration is longer for the older adults. Second, the perceptual identification times are longer for the older adults. Third, the time to complete the unlocking of Task 2 is longer for the older adults.

This third finding indicates a specific type of task-coordination strategy difference just as we found with the SRD models of Experiment 1. However, in the RSL models of Experiment 2, the strategy difference is much smaller than before. In particular, the time spent unlocking Task 2 during trials of Experiment 1 was about 2.5 cognitive-processor cycles (approximately $140 \mathrm{~ms}$ ) longer for the older adults than the young adults. The time spent unlocking Task 2 during trials of Experiment 2 was about 1.4 cognitive-processor cycles $(78 \mathrm{~ms})$ longer for the older adults than for the young adults. Thus, this difference in strategy between the young and older adults is smaller in Experiment 2.
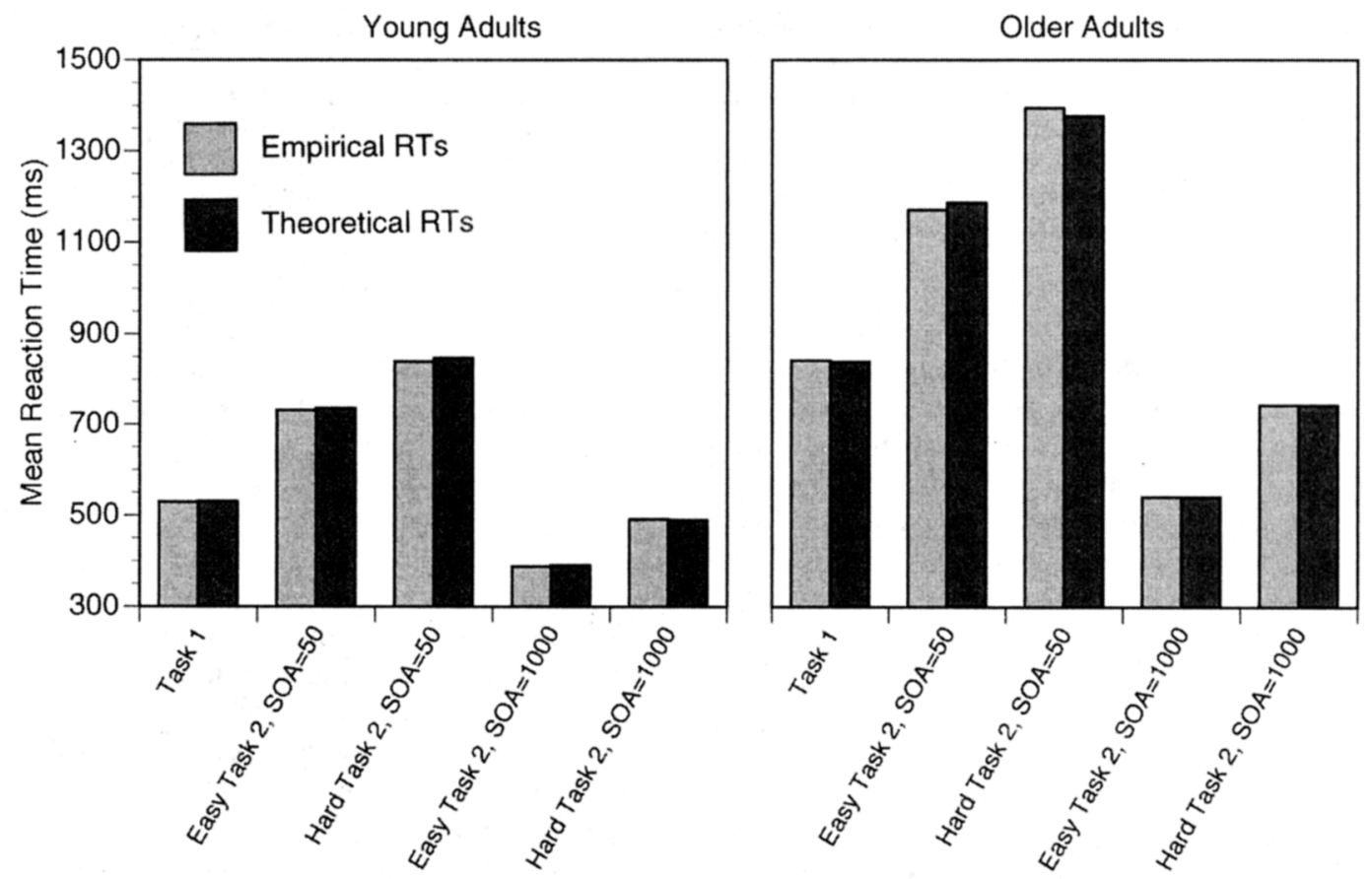

Figure 9. Empirical and theoretical mean reaction times (RTs) from the best fitting response-selection lockout models for Experiment 2. The panel on the left depicts RTs for the young adults (root-mean-square error [RMSE] $=4.3$ ). The panel on the right depicts RTs for the older adults (RMSE $=11.3$ ). SOA $=$ stimulus onset asynchrony. 
Table 5

Parameter Values for Best-Fitting Response-Selection Lockout Models as a Function of Age Group and Task Type

\begin{tabular}{|c|c|c|c|c|c|c|}
\hline \multirow[b]{2}{*}{ Parameter name and symbol } & \multicolumn{3}{|c|}{ Young adults } & \multicolumn{3}{|c|}{ Older adults } \\
\hline & T1 & $\mathrm{T} 2 \mathrm{E}$ & $\mathrm{T} 2 \mathrm{H}$ & $\mathrm{T} 1$ & T2E & $\mathrm{T} 2 \mathrm{H}$ \\
\hline \\
\hline $\begin{array}{l}t_{c} \\
\text { Working-memory gating time }\end{array}$ & 50 & 50 & 50 & 56 & 56 & 56 \\
\hline$\underset{\text { Stimulus identification time }}{\mathrm{t}_{\mathrm{g}}}$ & 25 & 25 & 25 & 28 & 28 & 28 \\
\hline $\begin{array}{l}t_{i} \\
\text { No. of movement features }\end{array}$ & 270 & 124 & 124 & 550 & 258 & 258 \\
\hline $\begin{array}{l}n_{f} \\
\text { Movement production time }\end{array}$ & 1 & 2 & 2 & 1 & 2 & 2 \\
\hline $\begin{array}{l}\text { No. of selection cycles } \\
\text { No }\end{array}$ & 100 & 150 & 150 & 112 & 168 & 168 \\
\hline $\begin{array}{l}\mathrm{n}_{\mathrm{s}} \\
\text { Response-selection time }\end{array}$ & 2.5 & 1.5 & 3.75 & 2.5 & 1.5 & 4.9 \\
\hline $\begin{array}{l}\mathrm{t}_{\mathrm{s}} \\
\text { Unlocking-onset latency }\end{array}$ & 125 & 75 & 187 & 140 & 84 & 273 \\
\hline $\begin{array}{l}t_{u} \\
\text { Minimum unlocking duration }\end{array}$ & 80 & 80 & 80 & 90 & 90 & 90 \\
\hline $\begin{array}{l}t_{v} \\
\text { Response-transduction time }\end{array}$ & 100 & 100 & 100 & 168 & 168 & 168 \\
\hline$t_{t}$ & 10 & 10 & 10 & 10 & 10 & 10 \\
\hline
\end{tabular}

Note. $\quad \mathrm{T} 1=$ Task $1 ; \mathrm{T} 2 \mathrm{E}=$ easy Task $2 ; \mathrm{T} 2 \mathrm{H}=$ hard Task 2.

To test the generalized slowing hypothesis, we assessed two other RSL models of the older adults' PRP data. In each model, it was assumed that the only difference between the age groups was the cognitive-processor cycle duration. In the first of these models, a cycle duration of $56 \mathrm{~ms}$ was used, and in the second model, a cycle duration of $75 \mathrm{~ms}$ was used. The parameter values for these models are shown in Table 6, and the theoretical RTs are shown in Figure 10. The RSL model with a cognitive-processor cycle duration of $56 \mathrm{~ms}$ underestimates all of the data points (RMSE = $261.7 \mathrm{~ms}$ ). The model with the $75 \mathrm{~ms}$ cognitive-processor cycle duration fares better, but it is still less accurate (RMSE $=32 \mathrm{~ms}$ ) than the previous best fitting RSL model in which age-related differences in perceptual identification and unlocking duration, as well as cognitive-processor cycle duration, were included (cf. Figure 9).

\section{Discussion}

The most important result from Experiment 2 is that when confronted with a harder primary task, both the young and older adults had additive PRP curves (i.e., there was no SOA $\times$ Task 2 Difficulty interaction). This suggests that each age group adapted flexibly to this change of dual-task context by using a taskcoordination strategy with nonoverlapping response-selection stages (unlike in Experiment 1). Thus, the results from each age group can be successfully fit by an RSL model in which Task 2 processing is locked out before Task 2 response selection begins. Young and older adults also used similar Task 2 unlocking strategies during Experiment 2.

Nevertheless, during Experiment 2, the older adults again were slower and had a larger PRP effect than did the young adults. The sources of the larger PRP effect included the longer Task 1 perceptual identification times and the longer cognitive-processor cycle duration for older adults. The older adults' Task 2 unlocking times also contributed to the larger PRP effect, but to a lesser extent than in Experiment 1. Instead, in Experiment 2, the most important contributor to the size of the older adults' PRP effect is the long Task 1 perceptual identification time $(550 \mathrm{~ms}$ for the older adults vs. $270 \mathrm{~ms}$ for the young adults).

The RSL model with small age-related strategy differences and process-specific slowing of perceptual identification in addition to generalized slowing provides a better fit than does an RSL model with only a larger contribution of generalized slowing. Thus, the effects of age on performance in Experiment 2 cannot be attributed to a single factor.

Experiments 1 and 2 together demonstrate that both older and young adults can exert flexible control over their task-coordination strategies, because both age groups chose a late Task 2 lockout point in Experiment 1 and an early Task 2 lockout point in Experiment 2. Furthermore, in Experiment 2, differences in taskcoordination strategies were not as important as process-specific slowing for explaining age-related performance in the PRP procedure.

\section{General Discussion}

The results from our PRP experiments and various types of AEC models show that age-related declines in dual-task performance are not unitary. Instead, we found that differences in taskcoordination strategies, process-specific slowing, and generalized slowing all contributed to older adults' PRP performance. For example, compared with young adults, older adults had a disproportionately larger PRP effect in Experiment 1. This disproportionate effect was much less pronounced in Experiment 2. The 
Table 6

Parameter Values for Two Generalized Slowing Response-Selection Lockout Models as a Function of Task Type

\begin{tabular}{|c|c|c|c|c|c|c|}
\hline \multirow[b]{2}{*}{ Parameter name and symbol } & \multicolumn{3}{|c|}{$12 \%$ slowing } & \multicolumn{3}{|c|}{$50 \%$ slowing } \\
\hline & $\mathrm{T} 1$ & T2E & $\mathbf{T} 2 \mathbf{H}$ & $\mathrm{T} 1$ & $\mathrm{~T} 2 \mathrm{E}$ & $\mathrm{T} 2 \mathrm{H}$ \\
\hline \multicolumn{7}{|l|}{ Cycle duration } \\
\hline $\begin{array}{l}t_{c} \\
\text { Working-memory gating time }\end{array}$ & 56 & 56 & 56 & 75 & 75 & 75 \\
\hline$\stackrel{\mathrm{t}_{\mathrm{g}}}{\text { Stimulus identification time }}$ & 28 & 28 & 28 & 38 & 38 & 38 \\
\hline $\begin{array}{l}\mathrm{t}_{\mathrm{i}} \\
\text { No. of movement features }\end{array}$ & 302 & 139 & 139 & 405 & 186 & 186 \\
\hline $\begin{array}{l}\mathbf{n}_{\mathbf{f}} \\
\text { Movement production time }\end{array}$ & 1 & 2 & 2 & 1 & 2 & 2 \\
\hline No. of selection cycles & 112 & 168 & 168 & 150 & 225 & 225 \\
\hline $\begin{array}{l}\quad \mathrm{n}_{\mathrm{s}} \\
\text { Response-selection time }\end{array}$ & 2.5 & 1.5 & 3.75 & 2.5 & 1.5 & 3.75 \\
\hline $\begin{array}{c}\mathrm{t}_{\mathrm{s}} \\
\text { Unlocking-onset latency }\end{array}$ & 140 & 84 & 210 & 188 & 113 & 281 \\
\hline${ }_{\text {Minimum unlocking duration }}^{t_{u}}$ & 90 & 90 & 90 & 120 & 120 & 120 \\
\hline $\begin{array}{l}\mathrm{t}_{\mathrm{v}} \\
\text { Suspension waiting time }\end{array}$ & 112 & 112 & 112 & 150 & 150 & 150 \\
\hline $\begin{array}{c}t_{w} \\
\text { Response-transduction time }\end{array}$ & 0 & 0 & 0 & 0 & 0 & 0 \\
\hline$t_{T}$ & 10 & 10 & 10 & 10 & 10 & 10 \\
\hline
\end{tabular}

Note. $\mathrm{T} 1=$ Task $1 ; \mathrm{T} 2 \mathrm{E}=$ easy Task $2 ; \mathrm{T} 2 \mathrm{H}=$ hard Task 2 .

different results from the two experiments can be explained by differences in task-coordination strategies. In Experiment 1, the older adults took much longer to unlock Task 2 than did the young adults. However, in Experiment 2, Task 2 unlocking durations were quite similar for both age groups. When the unlocking durations are similar for both age groups, the size of the PRP effect for older adults is more proportional to the young adults.

We also found that process-specific slowing contributed crucially to the model of the older adults' data. Perceptual identification times were longer for the older adults, and this occurred in addition to an increase of the cognitive-processor cycle duration. Our results agree with those of others who have found evidence for process-specific slowing (e.g., Bashore, Van der Molen, Ridderinkhof, \& Wylie, 1997; Strayer, Wickens, \& Braune, 1987).

Given these findings, we may now re-examine some previous explanations for the effects of aging on dual-task performance. The first is the generalized slowing hypothesis. As mentioned before, our results are consistent with the existence of generalized slowing, but this is not enough to explain the PRP effects from the present experiments. Instead, generalized slowing must be combined with differences in task-coordination strategy and processspecific slowing. Therefore, we reject a pure version of the generalized-slowing hypothesis, where generalized slowing is the only factor affecting the performance of older adults.

Related to the generalized-slowing hypothesis is the complexity hypothesis (McDowd \& Craik, 1988; McDowd et al., 1991). According to it, age differences in performance increase as the complexity (or number of steps to be performed) increases. If so, then larger effects of aging that occur in dual-task situations would stem from increased complexity in those situations. However, the present results are inconsistent with the complexity hypothesis. When complexity was increased from Experiment 1 to Experiment 2 (Task 1 changed from a two-choice to a four-choice RT task), the difference between the young and older adults' taskcoordination strategies (and the sizes of their PRP effects) actually decreased, rather than increased. Other researchers have also reported results opposed to the complexity hypothesis (e.g., Crossley \& Hiscock, 1992; Korteling, 1991).

Another explanation for age-related declines in dual-task performance is the reduced-resource hypothesis (Craik \& Byrd, 1982). We consider it separately here, although it is often implicit in other explanations of how aging affects dual-task performance. For example, information-processing speed is sometimes construed as the diminished resource (Salthouse, 1985, 1996). According to the reduced-resource hypothesis, older adults have reduced cognitive resources. Differences in performance between young and older adults are supposedly largest in situations that make the most demands for the resource. Performing two tasks at once is assumed to be more resource demanding than performing one task, and therefore age differences are larger in dual-task situations.

Regarding this latter assumption, the present results are not consistent with the reduced-resource hypothesis. Because Task 1 in Experiment 2 was harder than Task 1 in Experiment 1, the diminished-resource hypothesis would predict larger age effects in Experiment 2 than in Experiment 1. Yet we found that the relative size of PRP effect for older adults was larger in Experiment 1 than Experiment 2. Furthermore, the EPIC archi- 
Empirical RTs

Theoretical RTs, $12 \%$ Slowing

Theoretical RTs, 50\% Slowing

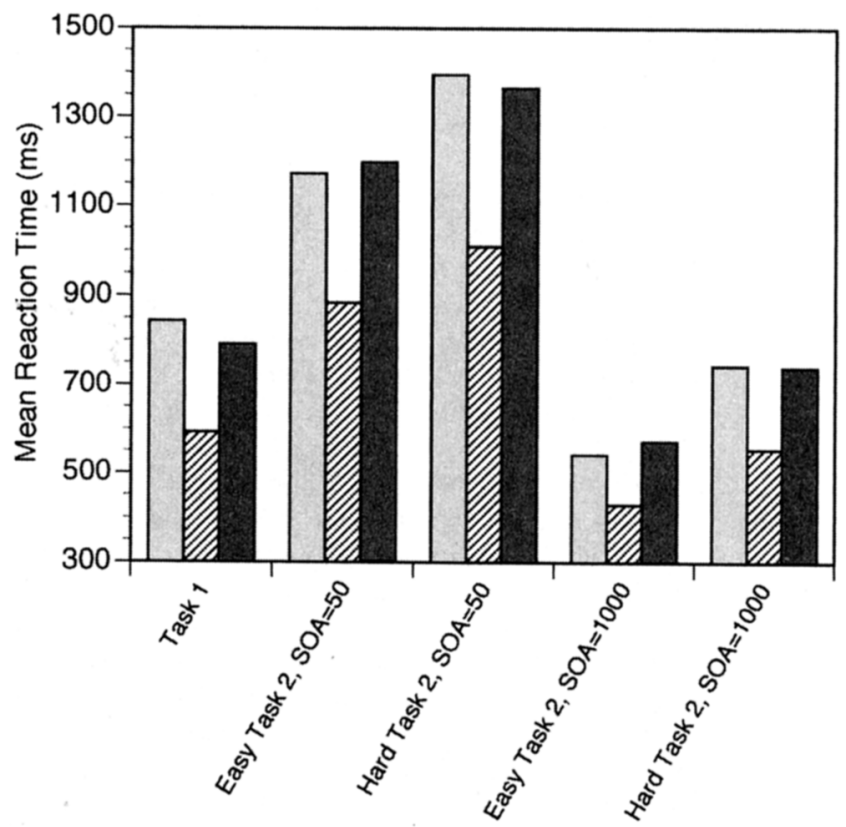

Figure 10. Empirical and theoretical mean reaction times (RTs) from two additional response-selection lockout models that tested the generalizedslowing hypothesis for the older adults in Experiment 2. When the cognitive-processor cycle duration was $56 \mathrm{~ms}$ (12\% slowing), root-meansquare error $(\mathrm{RMSE})=261.7$. When the cognitive-processor cycle duration was $75 \mathrm{~ms}$ (50\% slowing), RMSE $=31.92$. Neither of these models provides as good a fit to the data as the one whose theoretical RTs are depicted in Figure 9. SOA = stimulus onset asynchrony.

tecture and its AEC models provide a good fit of the PRP data from the present experiments, even though our theoretical framework has no structural cognitive bottlenecks that would limit processing capacity (Meyer \& Kieras, 1997a, 1999). In addition, other data from the PRP procedure with young adults (Pashler, 1994; Pashler \& Johnston, 1989) and older adults (Hartley \& Little, 1999) have been used successfully to argue against resource theories of dual-task performance.

Also relevant to age effects on dual-task performance are explanations based on declining frontal-lobe or executivecontrol functions (Moscovitch \& Winocur, 1992, 1995; West, 1996). For example, Allen et al. (1998) found larger PRP effects in older adults and inferred that they have a deficit in time-sharing ability. However, according to our AEC models, large PRP effects are not the hallmark of decreased or deficient executive control per se. To comply with PRP task instructions, participants often must postpone their Task 2 responses (Meyer \& Kieras, 1997b; Schumacher et al., 1999). Exactly how they implement the postponement is determined by their task- coordination strategy, and the strategy helps determine the size of the PRP effect. Thus, the claim that older adults have diminished executive (or time sharing) ability cannot be supported by appeal to large PRP effects.

Instead, our AEC models make different predictions about what may happen when there is a lack of executive control over the two tasks in a PRP experiment. Under such conditions, there would be much overlap in the processing stages for the two tasks, because lack of executive control would lead to the Task 2 lockout point and the Task 1 unlocking event not being set. The increased overlap would result in much smaller PRP effects. In addition, there would be frequent out-of-order responses, especially at the shortest SOA, and when Task 1 responses are slow in comparison to Task 2 responses.

However, we found none of these manifestations of poor executive control. Our older adult participants had larger but not enormous PRP effects compared to young adult participants. In Experiment 2, both young and older adults had additive PRP curves, indicating they were able to enforce an early Task 2 lockout point. There were very few out-of-order responses by either age group, again indicating that older adults were able to effectively enforce executive control for complying with the PRP instructions. Other researchers have reached a similar conclusion regarding the intact ability of older adults to exert executive control over dual-task performance (Hartley \& Little, 1999; Kramer, Larish, \& Strayer, 1995; Kramer, Larish, Weber, \& Bardell, 1999).

In conclusion, it appears that the effects of aging on dual-task performance are subtle and complex; task-coordination strategy differences across studies can explain many of the inconsistent findings in the aging and dual-task performance literature. To reach these conclusions, it is necessary to use a detailed analytical model, because several factors contribute to dual-task performance, including flexible task-coordination strategies. Detailed modeling of performance is needed even for the very basic tasks and relatively constrained PRP procedure used here. Without AEC models such as the EPIC architecture provides, it would be very difficult to understand the combined results of Experiment 1 (with disproportionately large PRP effects for the older adults) and Experiment 2 (with approximately proportionate PRP effects for the older adults). Our results and conclusions have important implications for researchers who use dual-task techniques to study the effects of aging on other cognitive abilities (e.g., working memory). These studies must take into account that larger dualtask costs for older adults can stem from strategic differences as well as process-specific slowing, rather than simply from diminished general capacities or resources.

\section{References}

Allen, P. A., Smith, A. F., Vires-Collins, H., \& Sperry, S. (1998). The psychological refractory period: Evidence for age differences in attentional time-sharing. Psychology and Aging, 13, 218-229.

Allport, D. A. (1993). Attention and control: Have we been asking the wrong questions? A critical review of 25 years. In D. E. Meyer \& S. Kornblum (Eds.), Attention and performance XIV: Synergies in experimental psychology, artificial intelligence, and cognitive neuroscience (pp. 183-218). Cambridge, MA: MIT Press. 
Anderson, J. R. (1993). Rules of the mind. Hillsdale, NJ: Erlbaum.

Anderson, N. D., Craik, F. I. M., \& Naveh-Benjamin, M. (1998). The attentional demands of encoding and retrieval in younger and older adults: 1. Evidence from divided attention costs. Psychology and Aging, 13, 405-423.

Baron, A., \& Matilla, W. R. (1989). Response slowing of older adults: Effects of time-limit contingencies on single- and dual-task performances. Psychology and Aging, 4, 66-72.

Bashore, T. R. (1994). Some thoughts on neurocognitive slowing. Acta Psychologica, 86, 295-325.

Bashore, T. R., Van der Molen, M. W., Ridderinkhof, K. R., \& Wylie, S. A. (1997). Is the age-complexity effect mediated by reductions in a general processing resource? Biological Psychology, 45, 263-282.

Cerella, J. (1985). Information processing rates in the elderly. Psychological Bulletin, 98, 67-83.

Craik, F. I. M. (1977). Age differences in human memory. In J. E. Birren \& K. W. Schaie (Eds.), Handbook of the psychology of aging (pp. 384-420). New York: Van Nostrand Reinhold.

Craik, F. I. M., \& Byrd, M. (1982). Aging and cognitive deficits: The role of attentional resources. In F. I. M. Craik \& A. S. Trehub (Eds.), Aging and cognitive processes (pp. 191-211). New York: Plenum.

Crossley, M., \& Hiscock, M. (1992). Age-related differences in concurrent-task performance of normal adults: Evidence for a decline in processing resources. Psychology and Aging, 7, 499-506.

Damos, D. L., \& Wickens, C. D. (1980). The identification and transfer of timesharing skills. Acta Psychologica, 46, 15-39.

De Jong, R. (1993). Multiple bottlenecks in overlapping task performance. Journal of Experimental Psychology: Human Perception and Performance, 19, 965-980.

Hartley, A. A. (1992). Attention. In F. I. M. Craik \& T. A. Salthouse (Eds.), The handbook of aging and cognition (pp. 3-49). Hillsdale, NJ: Erlbaum.

Hartley, A. A., \& Little, D. M. (1999). Age-related differences and similarities in dual-task interference. Journal of Experimental Psychology: General, 128, 416-449.

Hawkins, H. L., Kramer, A. F., \& Capaldi, D. (1992). Aging, exercise, and attention. Psychology and Aging, 7, 643-653.

Hawkins, H. L., Rodriguez, E., \& Reicher, G. M. (1979). Is time-sharing a general ability? (Office of Naval Research Tech. Rep. No. 3). Eugene: University of Oregon.

Ivry, R. B., Franz, E. A., Kingstone, A., \& Johnston, J. C. (1994, November). The PRP effect in a split-brain patient: Response uncoupling despite normal interference. Paper presented at the meeting of the Psychonomic Society, St. Louis, MO.

Karlin, L., \& Kestenbaum, R. (1968). Effects of number of alternatives on the psychological refractory period. Quarterly Journal of Experimental Psychology, 20, 67-178.

Kausler, D. H. (1991). Experimental psychology, cognition, and human aging (2nd ed.). New York: Springer-Verlag.

Korteling, J. (1991). Effects of skill integration and perceptual competition on age-related differences in dual-task performance. Human Factors, 33. $35-44$.

Kramer, A. F., \& Larish, J. L. (1996). Aging and dual-task performance. In W. A. Rogers (Ed.), Aging and skilled performance: Advances in theory and applications (pp. 83-112). Mahwah, NJ: Erlbaum.

Kramer, A. F., Larish, J. L., \& Strayer, D. (1995). Training for attention control in dual-task settings: A comparison of young and old adults. Joumal of Experimental Psychology: Applied, 1, 50-76.

Kramer, A. F., Larish, J. L., Weber, T. A., \& Bardell, L. (1999). Training for executive control: Task coordination strategies and aging. In D. Gopher \& A. Koriat (Eds.), Attention and performance XVII (pp. 617652). Cambridge, MA: MIT Press.

McCann, R. S., \& Johnston, J. C. (1992). Locus of the single-channel bottleneck in dual-task performance. Joumal of Experimental Psychology: Human Perception and Performance, 18, 471-484.

McDowd, J. M., \& Craik, F. I. M. (1988). Effects of aging and task difficulty on divided attention performance. Journal of Experimental Psychology: Human Perception and Performance, 14, 267-280.

McDowd, J. M., Vercruyssen, M., \& Birren, J. E. (1991). Aging, divided attention, and dual-task performance. In D. L. Damos (Ed.), Multiple task performance (pp. 387-414). Bristol, PA: Taylor \& Francis.

Meyer, D. E., \& Kieras, D. E. (1997a). A computational theory of executive cognitive processes and multiple-task performance: Part 1. Basic Mechanisms. Psychological Review, 104, 3-65.

Meyer, D. E., \& Kieras, D. E. (1997b). A computational theory of executive cognitive processes and multiple-task performance: Part 2. Accounts of psychological refractory-period phenomena. Psychological Review, 104, 749-791.

Meyer, D. E., \& Kieras, D. E. (1999). Précis to a practical unified theory of cognition and action: Some lessons from computational modeling of human multiple-task performance. In D. Gopher \& A. Koriat (Eds.), Attention and performance XVII (pp. 15-88). Cambridge, MA: MIT Press.

Meyer, D. E., Kieras, D. E., Lauber, E., Schumacher, E., Glass, J., Zurbriggen, E., Gmeindl, L., \& Apfelblat, D. (1995). Adaptive executive control: Flexible multiple-task performance without pervasive immutable response-selection bottlenecks. Acta Psychologica, 90, 163-190.

Moscovitch, M., \& Winocur, G. (1992). The neuropsychology of memory and aging. In F. I. M. Craik \& T. A. Salthouse (Eds.), The handbook of aging and cognition (pp. 315-372). Hillsdale, NJ: Erlbaum.

Moscovitch, M., \& Winocur, G. (1995). Frontal lobes, memory and aging. In J. Grafman, K. J. Holyoak, \& F. Boller (Eds.), Annals of the New York Academy of Sciences: Vol. 769. Structure and functions of the human prefrontal cortex (pp. 119-150). New York: New York Academy of Sciences.

Navon, D. (1984). Resources-A theoretical soup stone'? Psychological Review, 91, 216-234.

Newell, A. (1990). Unified theories of cognition. Cambridge, MA: Harvard University Press.

Park, D. C., Smith, A. D., Dudley, W. N., \& Lafronza, V. (1989). The effects of age and a divided attention task presented during encoding and retrieval on memory. Journal of Experimental Psychology: Learning, Memory, and Cognition, 15, 1185-1191.

Pashler, H. (1984). Processing stages in overlapping tasks: Evidence for a central bottleneck. Journal of Experimental Psychology: Human Perception and Performance, 10, 358-377.

Pashler, H. (1994). Dual-task interference in simple tasks: Data and theory. Psychological Bulletin, 116, 220-244.

Pashler, H., \& Johnston, J. C. (1989). Chronometric evidence for central postponement in temporally overlapping tasks. Quarterly Joumal of Experimental Psychology: Human Experimental Psychology, 4, 19-45.

Ponds, R. W., Brouwer, W. H., \& van Wolffelaar, P. C. (1988). Age differences in divided attention in a simulated driving task. Journals of Gerontology, 43, P151-P156.

Salthouse, T. A. (1985). Speed of behavior and its implications for cognition. In J. E. Birren \& K. W. Schaie (Eds.), Handbook of the psychology of aging (pp. 400-426). New York: Van Nostran Reinhold.

Salthouse, T. A. (1996). The processing-speed theory of adult age differences in cognition. Psychological Review, 103, 403-428.

Salthouse, T. A., \& Babcock, R. L. (1991). Decomposing adult age differences in working memory. Developmental Psychology, 27, 763776 .

Salthouse, T. A., Fristoe, N. M., Lineweaver, T. T., \& Coon, V. E. (1995). Aging of attention: Does the ability to divide decline? Memory \& Cognition, 23, 59-71.

Salthouse, T. A., Rogan, J. D., \& Prill, K. A. (1984). Division of attention: 
Age differences on a visually presented memory task. Memory \& Cog. nition, 12, 613-620.

Schumacher, E. H., Lauber, E. J., Glass, J. M., Zurbriggen, E., Gmeindl, L., Kieras, D. E., \& Meyer, D. E. (1999). Concurrent response-selection processes in dual-task performance: Evidence for adaptive executive control over task-scheduling strategies. Journal of Experimental Psychology: Human Perception and Performance, 25, 791-814.

Schumacher, E. H., Seymour, T. L., Glass, J. M., Lauber, E. J., Kieras, D. E., \& Meyer, D. E. (1997, November). Virtually perfect time sharing in dual-task performance. Paper presented at the meeting of the Psychonomic Society, Philadelphia, PA.

Smith, G. A., \& Brewer, N. (1995). Slowness and age: Speed-accuracy mechanisms. Psychology and Aging, 10, 238-247.

Somberg, B. L., \& Salthouse, T. A. (1982). Divided attention abilities in young and old adults. Journal of Experimental Psychology: Human Perception and Performance, 8, 651-663.
Strayer, D. L., Wickens, C. D., \& Braune, R. (1987). Adult age differences in the speed and capacity of information processing: 2 . An electrophysiological approach. Psychology and Aging, 2, 99-110.

Welford, A. T. (1952). The 'psychological refractory period' and the timing of high-speed performance-A review and a theory. British Journal of Psychology, 43, 2-19.

Welford, A. T. (1958). Aging and human skill. London: Oxford University Press.

West, R. L. (1996). An application of prefrontal cortex function theory to cognitive aging. Psychological Bulletin, 120, 272-292.

Woodruff, D. S. (1975). Relationships among EEG alpha frequency, reaction time, and age: A biofeedback study. Psychophysiology, 12, 673681.

Woodruff-Pak, D. S. (1997). The neuropsychology of aging. Malden, MA: Blackwell Publishers.

\section{Appendix}

\section{Parameters Associated With the Adaptive Executive-Control Models}

Several types of parameters relevant to the executive-process/ interactive-control (EPIC) architecture and various adaptive executivecontrol (AEC) models are summarized in Table 1 and are explained below. The values of these parameters for the strategic response-deferment (SRD) models from Experiment 1 are shown in Table 2 (the models of best fit for both young and older adults) and Table 3 (the generalized-slowing SRD models). Likewise, the parameter values for the RSL models from Experiment 2 are shown in Table 4 (the models of best fit for both young and older adults) and Table 5 (the generalized-slowing response-selection lockout [RSL] models).

\section{Cognitive-Processor Parameters}

The most basic parameter associated with EPIC's cognitive processor is the cycle duration $\left(t_{c}\right)$. The number of production rules that have to be processed in a given cycle does not affect $t_{c}$. However, because individual task and executive processes typically take more than one cycle to be completed, their completion times and resulting RTs depend directly on $t_{c}$. For young adults the standard value of $t_{c}(50 \mathrm{~ms})$ was used. For the older adults, we set $t_{c}$ to be $12 \%\left(56 \mathrm{~ms}\right.$ ) larger than the young adult $t_{c}$ (see text for justification). We also tested some models that involved a larger increase $(50 \% ; 75 \mathrm{~ms})$ in the older adult $\mathrm{t}_{\mathrm{c}}$.

Stemming from the cognitive-processor cycle duration is another parameter, the working-memory gating time $\left(t_{g}\right)$. It is the time between the moments when a new item of information enters working memory and the cognitive processor can first use this item in subsequent operations. On average, $t_{g}$ equals half of $t_{c}$, because the cognitive processor examines the contents of working memory at the start of each cycle.

\section{Perceptual-Processor Parameters}

For present purposes, one perceptual-processor parameter is important, the stimulus identification time $\left(t_{i}\right)$. It is the time from the onset of a presented stimulus until the perceptual processor for its modality puts the identity of the stimulus in working memory. The value of $t_{i}$ for Task 1 can be derived from Equation $I$ (the reaction time [RT] equations are described in the next section) by inserting the parameter values for $t_{g}, t_{s}, t_{m}$, and $t_{r}$ and solving for the value of $t_{i}$. The value of $t_{i}$ for Task 2 can be derived from Equation 4 in a similar fashion. Therefore, $t_{\mathrm{i}}$ is a data-derived parameter.

\section{Motor-Processor Parameters}

Several parameters contribute to operations by EPIC's motor processors. They include (a) the number of movement features, $n_{f}$, prepared by a motor processor when it converts a selected response symbol to an overt movement; (b) the time per movement feature, $t_{f}$, taken to complete this conversion $\left(t_{f}\right.$ corresponds with $\left.t_{c}\right)$; and (c) the action initiation time, $t_{a}$, taken to begin an overt movement after all of its requisite features have been prepared (note that $t_{a}=$ duration of one cognitive processor cycle). The parameters combine to yield a movement production time $\left(t_{m}\right)$. By definition, $t_{m}=\left(n_{\mathrm{f}} \times t_{\mathrm{f}}\right)+t_{\mathrm{a}}$.

The features that are used by the manual motor processor include the type of movement to make (in this case, a button press), the hand to use, and the finger to use. For Task 1, the type of movement and the hand are preset (because all movements are button presses, and the left hand is always used for Task 1, the participant can select these in advance of the stimulus presentation). Therefore, only one feature need be selected for Task 1, which is the finger to use. In contrast, participants cannot preset the hand feature for Task 2, therefore Task 2 involves two feature selections, which are the hand to use and the finger to use. Because we are able to specify in advance the number of manual motor features for each task, $t_{m}$ is a predetermined parameter.

\section{Task-Process Parameters}

For each task process in an AEC model, an important parameter is the number of response-selection cycles $\left(n_{s}\right)$ per trial. It equals the mean total cycles taken by EPIC's cognitive processor in selecting the response to a 
stimulus. The value of $n_{\mathrm{s}}$ depends on the specific production rules used during response selection, which may change as a function of factors such as $S-R$ compatibility and S-R numerosity. The number of responseselection cycles combines multiplicatively with the cycle duration, to yield the response-selection time $\left(t_{s}=n_{s} \times t_{c}\right)$.

For the two-choice Task 1 used in Experiment 1, the production rules of response selection that are specified in the SRD model first check the contents of working memory for one particular tone (e.g., the low tone). If this provides a match, then the appropriate response code is added to working memory and response selection is complete. If there is no match, then on the next response-selection cycle, the contents of working memory are checked for the other tone (e.g., the high tone). Hence, there is an average of 1.5 response-selection cycles for the two-choice Task 1 . For the four-choice Task 1 in Experiment 2, response selection may take as many as four cycles to complete, therefore the average number of responseselection cycles is 2.5 . Similar logic is used to derive an average of 1.5 response-selection cycles for the easy Task 2.

Quantifying the number of response-selection cycles for the hard Task 2 is more complicated, because there are several plausible response-selection strategies. For example, the most straightforward strategy involves checking the contents of working memory for each digit in turn. If so, then with eight digits, response selection takes an average of 4.5 cycles. However, there are other response-selection strategies that yield different averages, such as checking for all of the digits mapped to the index finger first. If none match the contents of working memory, then on the next responseselection cycle, the response code for the middle finger can be added to working memory. This strategy yields an average of three responseselection cycles. Because there is no way to determine a priori which response-selection strategy participants will use, we estimated the number of response-selection cycles for the hard Task 2 from its mean RT at the longest SOA. This value can be derived from Equation 4 by inserting the other parameters and solving for $t_{\mathrm{s}}$. Thus, the number of response-selection cycles, and thereby the value of $t_{s}$ can be predetermined for Task 1 and for the easy Task 2, but is data derived for the hard Task 2 .

\section{Executive-Process Parameters}

For our purposes, two more parameters are associated with the executive process of the AEC models. The first of these is the unlocking-onset latency $\left(t_{u}\right)$. It is the time between two intermediate events: (a) transmission of a selected Task 1 response to its motor processor, and (b) initiation of the shift from deferred to immediate response-transmission mode for the Task 2 production rules. The value of $t_{\mathrm{u}}$ is set by specifying what internal state during the production of an overt Task 1 response qualifies Task 1 to be declared "done." This specification may depend on several factors, such as which motor processor is used for performing each task of the PRP procedure, and how conservative the executive process must be to ensure that Task 1 responses always precede Task 2 responses. The second executive-process parameter is the minimum unlocking duration $\left(t_{v}\right)$. Its value is set by specifying the production rules that unlock Task 2 after Task 1 has been declared "done."

\section{Degrees of Freedom}

Although the total number of parameters in the AEC models may seem large, this appearance is deceptive. Many of the parameters are linearly or multiplicatively related to each other; we treat them as being distinct merely for purposes of exposition. Furthermore, the mean numerical values assigned to many of these parameters are predetermined and are not changed in order to fit the data. Thus, the model actually has relatively few adjustable parameters and degrees of freedom with which to account for empirical data. The source for each parameter value is indicated in Table 1 as either predetermined $(\mathrm{P})$ or derived from the data $(\mathrm{D})$. It can be seen from Table 1 that five of the parameters are free to vary, but this actually translates to four free parameters, because response-selection time is a product of the number of selection cycles and the cognitive-processor cycle duration. Thus, we used four free parameters to estimate 4 data points.

Some readers may object to this, pointing out that we have not provided a true test of the EPIC architecture and the AEC models. However, it should be noted that our intention here is not to test the AEC models- that task has already been completed by Meyer and Kieras (1997a, 1997b) with data sets that afforded a conservative low ratio of free parameters to degrees of freedom in the data. Furthermore, the predictions of the AEC models have been empirically tested and born out (Meyer et al., 1995; Schumacher et al., 1997, 1999). Instead, our intention was to use an already well-validated model to interpret age differences in dual-task performance. Thus, our present emphasis is not on the overall fit of the model, but on how this fit was achieved for the young and older adults, respectively. To this end, the present parameter values represent unique solutions for best fitting the data, and their relative values provide important insights about cognitive aging effects on dual-task performance.

\section{Reaction Time Equations for the SRD and RSL Models}

Using the parameters described above, algebraic equations have been formulated for the theoretical mean RTs implied by the SRD and RSL models. With these equations, we can estimate appropriate values of some parameters on which the model and the EPIC architecture rely, and we can evaluate the model's goodness of fit to empirical data in a principled fashion.

\section{Task 1 Equation}

According to the SRD and RSL models, Task 1 of the PRP procedure receives highest priority, and performance of it progresses from start to finish in the same rapid fashion regardless of the stimulus-onset asynchrony (SOA) between the Task 1 and Task 2 stimuli. The model assumes that Task 1 entails a sequence of stages, which start at the onset of the Task 1 stimulus and include the following steps: (a) identification of the Task 1 stimulus by the auditory perceptual processor; (b) selection of a Task 1 response by the cognitive processor, and transmission of the response's identity to its motor processor; (c) preparation of movement features and initiation of action by the motor processor; and (d) transduction of the response movement. Thus, for Task 1, the theoretical RT on each trial of the PRP procedure is

$$
R T 1=t_{i 1}+t_{g}+t_{s 1}+t_{m 1}+t_{r 1} .
$$

Here $t_{i 1}$ is the Task 1 stimulus-identification time; $t_{g}$ is the workingmemory gating time; $t_{81}$ is the average Task 1 response-selection time; $t_{m 1}$ is the Task 1 movement-production time; and $\mathrm{t}_{\mathrm{r} 1}$ is the Task 1 responsetransduction time (i.e., the time from actual depression of the response key until the computer registers a response).

Some further aspects of Equation 1 should be mentioned as well. According to it, Task 1 RTs are independent of the SOA and Task 2 response-selection difficulty. Consistent with typical instructions for the PRP procedure, this independence occurs because the executive process of the SRD model always gives highest priority to Task 1 .

\section{Task 2 Equations for the SRD Model}

Unlike the case for Task 1 RTs, the SRD model implies that theoretical Task 2 RTs embody effects of both the SOA and Task 2 response-selection difficulty. The expected pattern stems from properties of the model's executive process. In particular, under the SRD model, there are five alternative paths of processing that may lead from Task 2 stimuli to Task 2 responses. The path that is actually taken during an individual trial depends on the SOA, the stimulus-identification times, and the response-selection times in Tasks 1 and 2. For each possible path, a distinct equation 
characterizes the theoretical Task $2 \mathrm{RT}$ as a function of the SRD model's parameters and SOA. The SOA is especially important here because it determines whether the difficulty of response selection in Task 2 contributes additively or interactively to the Task 2 RT. We are concerned with only three of these paths, because they are the ones necessary for modeling performance at the shortest and longest SOAs in Experiment 1.

Path 1 (SRD model): $R T$ for Task 2 with postselection slack. In order for Task 2 processing to follow Path 1, the SOA must be very short and satisfy the following constraint defined by the parameters of the SRD model:

$$
S O A \leq t_{i 1}+t_{s 1}+t_{u}-S O A-t_{i 2}-t_{32} .
$$

Here $t_{11}$ and $t_{s 1}$ are again respectively the Task 1 stimulus-identification and response-selection times; $t_{u}$ is the unlocking-onset latency of the executive process; $t_{22}$ and $t_{32}$ are respectively the Task 2 stimulus-identification and response-selection times.

When processing for Task 2 follows Path 1 , the Task 2 stimulus occurs sufficiently early that a selected Task 2 response code is sent to working memory before the executive process starts unlocking Task 2. Further progress on Task 2 therefore has to wait until Task 1 is declared "done" and the executive process finishes unlocking Task 2 , which permits the selected Task 2 response to be sent from working memory to its motor processor for movement preparation and overt action. Thus, taking Path 1 introduces postselection slack (i.e., a pause after Task 2 response selection). When there is postselection slack, the Task 2 stimulus-identification and response-selection times do not contribute to the Task $2 \mathrm{RT}$; the slack absorbs them. Instead, the Task 2 RT includes additive contributions from several other sources: (a) the time that the SRD model's executive process takes to finish unlocking Task $2\left(t_{i 1}+t_{g}+t_{s 1}+t_{u}+t_{v}\right)$; (b) the Task 2 movement-production time $\left(t_{\mathrm{m}_{2}}\right)$; and (c) the Task 2 response-transduction time $\left(t_{\mathrm{r}}\right)$. Combining these contributions and subtracting the SOA yields an equation for Task 2 RT along Path 1:

RT2(SRD: Path 1) $=t_{i 1}+t_{g}+t_{s 1}+t_{u}+t_{v}+t_{m 2}+t_{r}-$ SOA.

The Task 2 RT equation for Path 1 has some interesting consequences. Because it omits the Task 2 response-selection time, the difficulty of response selection in Task 2 may not affect Task 2 RTs at very short SOAs.

However, Path 1 is not the only processing path that is possible for Task 2 when the SOA is short. On some subset of trials, the constraint imposed by inequality 1 will not be satisfied if the Task 2 stimulus identification or response-selection times are long (or Task 1 stimulusidentification and response-selection times are short). On these occasions, processing for Task 2 will follow a different path.

Path 2 (SRD model): RT for Task 2 with midselection slack. When Task 2 processes follow Path 2, the response selection for Task 2 again starts before Task 1 is declared "done" and the executive process unlocks Task 2. However, Task 2 response selection does not finish until after the executive process has completed the unlocking phase; therefore, taking Path 2 introduces midselection slack (i.e., a pause during response selection) within Task 2.

When there is midselection slack, several components contribute additively to the Task 2 RT. These components include the following: (a) the time taken to identify the Task 2 stimulus, (b) start and progress part way through selecting a response, (c) unlock Task 2, (d) finish selecting the Task 2 response, (e) produce the response movement, and (f) transduce the overt response. Thus, the Task 2 RT obtained from Path 2 is

$$
\text { RT2(SRD: Path 2) }=t_{i 2}+t_{g}+t_{s 2}+t_{v}+t_{m 2}+t_{r} \text {. }
$$

In order to estimate mean RTs at the shortest $(50 \mathrm{~ms}) \mathrm{SOA}$, it is sometimes necessary to combine the estimates from Path 1 and Path 2 . Consider the young adults' SRD model first. We found that the inequality for Path 1 processing was satisfied for all combinations of $t_{s 1}$ and $t_{s 2}$ when Task 2 was easy. This is because response selection for Task 1 can be completed in either one or two response-selection cycles. Likewise, response selection for the easy Task 2 can be completed in either one or two response-selection cycles. It follows that there are four combinations of the number of response-selection cycles for Task 1 and the number of response-selection cycles for the easy Task 2 . Sometimes both Task 1 and Task 2 responses are selected with only one response-selection cycle. Other times, the Task 1 response is selected with one response-selection cycle, whereas the Task 2 response needs two response-selection cycles, and so forth. For each of these combinations, the inequality for Path 1 is satisfied. This means that for the easy Task 2 under the SRD model, the RT equation for Path 1 always yields the theoretical mean RT for the easy Task 2.

In contrast, the inequality for Path 1 is not always satisfied when Task 2 is hard. This is because response selection for the hard Task 2 can potentially take more than two cycles to complete. For the young adults, Task 2 processing followed Path $136 \%$ of the time and followed Path 2 $64 \%$ of the time. Thus, the theoretical mean RT for the hard Task 2 reflects a weighted average of contributions from Path 1 and Path 2.

A similar pattern was found with the older adults' SRD model. Like the young adults, the inequality for Path 1 was always satisfied when Task 2 was easy. When Task 2 was hard, processing took Path $144 \%$ of the time and Path $256 \%$ of the time. Thus, the theoretical mean RT for older adults when Task 2 was hard again reflects a weighted average of contributions from Path 1 and Path 2.

Path 4: RT for Task 2 at neutral baseline. The third path of interest here is one taken when SOA is moderately long. In this case, stimulus identification for Task 2 is not completed until after the executive process finishes unlocking and resuming Task 2 . As a result, no slack occurs within Task 2 when Path 4 is taken, because no Task 2 processes have to pause while the executive process unlocks Task 2 . Therefore, the RT equation for Path 4 is simply the sum of Task 2 stimulus identification, workingmemory gating, response selection, movement production, and response transduction times:

$$
\text { RT2(SRD: Path 4) }=t_{\mathrm{i} 2}+\mathrm{t}_{\mathrm{g}}+\mathrm{t}_{\mathrm{s} 2}+\mathrm{t}_{\mathrm{m} 2}+\mathrm{t}_{\mathrm{r}} \text {. }
$$

Task 2 Equation for the RSL Model. Under the RSL model, the RT equations for Task 1 and Task 2 at the longest SOA are the same as under the SRD model (Equations 1 and 5, respectively). However, the course of Task 2 processing when the SOA is short differs under the RSL model. In particular, the Task 2 lockout point is set to occur before the Task 2 response-selection stage. At the short SOA, there is only one processing path available, and Task 2 processing always pauses before response selection begins. Because there is no post-selection slack, the effect of Task 2 difficulty is not absorbed at short SOAs. Because of this, the effect of Task 2 response-selection difficulty is the same across SOAs. Thus, the RT equation for Task 2 at the short SOA is

RT2(RSL: short SOA) $=t_{i 1}+t_{g}+t_{s 1}+t_{u}+t_{\mathrm{v}}+t_{s 2}+t_{m 2}+t_{r}$.

Note. Sections of the Appendix are from "A Computational Theory of Executive Cognitive Processes and Multiple-Task Performance: Part 1. Basic Mechanisms," by D. E. Meyer and D. E. Kieras, 1997, Psychological Review, 104, pp. 3-65. Copyright 1997 by the American Psychological Association. Adapted with permission of the author.

Received August 25, 1998

Revision received August 26, 1999

Accepted October 19, 1999 